\title{
Computation of Whistler Ray Paths ${ }^{1}$
}

\author{
Irving Yabroff \\ Contribution From Stanford Research Institute, Menlo Park, Calif.
}

(Received May 8, 1961)

\begin{abstract}
Calculations of whistler ray paths in the outer ionosphere are shown for a variety of electron density profile models including exponential, constant, and columnar profiles. The Haselgrove formulation of the ray equations was used with the magneto-ionic representation of the wave refractive index to develop a set of differential equations for ray tracing suitable for inhomogeneous, anisotropic medium. The variation of paths with frequency, latitude, initial wave-normal angle, and other variables are examined for the purpose of providing a preliminary basis for comparison of the theoretical with some of the experimental results.
\end{abstract}

\section{Introduction}

Little work has previously been done to describe the mechanics and physics of electromagnetic wave propagation in an inhomogeneous anisotropic, medium. Of particular interest are certain phenomena taking place at frequencies below the plasma and gyrofrequencies of the ionosphere. The subject has become important during the last few years because of the increasing interest in VLF wave phenomena such as "whistlers."

Whistlers propagate in what is known as the "extraordinary" mode in magneto-ionic theory [Ratcliffe, 1959]. This mode of propagation is also described by the terms "whistler-mode" or "magneto-ionic duct." Details of the phenomena and additional references are given elsewhere [Helliwell and Morgan, 1953]. The basic theory of whistler propagation was first given by Storey [1953]; however, his description is valid only for a restricted set of conditions, many of which do not apply over the complete whistler path.

The work reported here is an attempt to demonstrate some of the complex phenomena of whistler propagation. The set of computations described here is in no sense complete or comprehensive; it is meant only to demonstrate a few interesting details of this mode of propagation and to stimulate further thought and work. Very little attempt has been made to interpret the form and shape of the results shown; such interpretations must arise from an examination of the form of the general solution of the ray path equations.

\section{Discussion}

\subsection{General Procedure}

Equations which are in a form useful for computation of ray paths in the ionosphere can be obtained from substitution of the familiar Appleton-Hartree expression for the complex refractive index into

1 Based on a report prepared by Stanford Research Institute for Stanford University under Prime Contract AF 18(603)-126 (Dec. 1959). ray equations derived by Haselgrove [1954]. The resulting equations in spherical coordinates $r, \theta, \phi$ are

$$
\begin{aligned}
\frac{d r}{d t} & =\frac{1}{\mu^{2}}\left(\rho_{r}-\mu \frac{\partial \mu}{\partial \rho_{r}}\right) \\
\frac{d \theta}{d t} & =\frac{1}{r \mu^{2}}\left(\rho_{\theta}-\mu \frac{\partial \mu}{\partial \rho_{\theta}}\right) \\
\frac{d \phi}{d t} & =\frac{1}{r \mu^{2} \sin \theta}\left(\rho_{\phi}-\mu \frac{\partial \mu}{\partial \rho_{\phi}}\right) \\
\frac{d \rho_{\tau}}{d t} & =\frac{1}{\mu} \frac{\partial \mu}{\partial r}+\rho_{\theta} \frac{d \theta}{d t}+\rho_{\phi} \frac{d \phi}{d t} \sin \theta \\
\frac{d \rho_{\theta}}{d t} & =\frac{1}{r}\left(\frac{1}{\mu} \frac{\partial \mu}{\partial \theta}-\rho_{\theta} \frac{d r}{d t}+r \rho_{\phi} \frac{d \phi}{d t} \cos \theta\right) \\
\frac{d \rho_{\phi}}{d t} & =\frac{1}{r \sin \theta}\left(\frac{1}{\mu} \frac{\partial \mu}{\partial \phi}-\rho_{\phi} \frac{d r}{d t} \sin \theta-r \rho_{\phi} \frac{d \theta}{d t} \cos \theta\right)
\end{aligned}
$$

where

$\mu=$ the real part of the complex phase refractive index,

$\rho_{r}, \rho_{\theta}, \quad \rho_{\phi}=$ the physical components of a vector of length $\mu$, and directed normal to the phase fronts (also called the wave normal or refractive index vector),

$t=$ time of phase travel along the ray (that is, $(f \Delta t) / c=$ number of wavelengths in the medium along the ray path),

$f=$ wave frequency,

$c=$ speed of light.

The quantity $\mu$ and its derivatives are calculated from the Appleton-Hartree formula as follows:

$$
\begin{aligned}
M^{2} & =(\mu-j K)^{2}=1-\frac{2 X(1-X-j Z)}{D} \\
D & =2(1-X-j Z)(1-j Z)-Y^{2} \sin ^{2} \psi+S \\
S & = \pm \sqrt{Y^{4} \sin ^{4} \psi+4 Y^{2}(1-X-j Z)^{2} \cos ^{2} \psi}
\end{aligned}
$$


where

$M=$ complex phase refractive index $=\mu-j k$,

$X=$ normalized electron density $=\left(N e^{2}\right) /\left(\omega^{2} m \epsilon_{0}\right)$,

$Y=$ normalized magnitude of the earth's magnetic field vector, $\vec{Y}=\left(\mu_{0} e \vec{H}_{e}\right) /(\omega m)$,

$Z=$ normalized collision frequency $=\left(\nu_{0}\right) / \omega$,

$\psi=$ angle between $\vec{\rho}$ and $\vec{Y}=\cos ^{-1}\left[\left(\rho_{r} Y_{r}+\rho_{\theta} Y_{\theta}\right.\right.$ $\left.\left.+\rho_{\phi} Y_{\phi}\right) /(\mu Y)\right]$,

$N=$ electron density,

$e=$ charge on an electron,

$m=$ mass of an electron,

$\omega=2 \pi f=2 \pi$. wave frequency,

$\epsilon_{0}=$ dielectric constant of free space,

$\mu_{0}=$ permeability of free space,

$\nu_{0}=$ collision frequency in collisions per second,

$K=$ imaginary part of the complex phase refractive index.

The two values of $M$ corresponding to the plus and minus sign on $S$ represent the two modes of ionospheric propagation commonly called the "ordinary" and "extraordinary" modes.

The derivatives of $\mu$ are

$$
\frac{\partial \mu}{\partial \rho_{i}}=\frac{\partial \mu}{\partial \psi} \frac{\partial \psi}{\partial \rho_{i}}=\frac{\partial \mu}{\partial \psi}\left(\frac{\rho_{i} Y \cos \psi-\mu Y_{i}}{\mu^{2} Y \sin \psi}\right)
$$

where $i=$ the coordinates $r, \theta, \phi$.

[Note: When $\psi \rightarrow 0, \partial \mu / \partial \psi \rightarrow 0, \quad \partial \psi / \partial \rho_{i} \rightarrow \infty$ but $\partial \mu / \partial \rho_{i} \rightarrow 0$.]

$$
\begin{aligned}
\frac{\partial \mu}{\partial \psi}= & \operatorname{Re} \frac{\partial M}{\partial \psi}=\operatorname{Re}\left(\frac{\left(M^{2}-1\right) Y^{2} \sin \psi \cos \psi}{M D}\right. \\
& \left.\left\{1-\frac{1}{S}\left[Y^{2} \sin ^{2} \psi-2(1-X-j Z)^{2}\right]\right\}\right)
\end{aligned}
$$

$$
\frac{\partial \mu}{\partial i}=\frac{\partial \mu}{\partial X} \frac{\partial X}{\partial i}+\frac{\partial \mu}{\partial Y} \frac{\partial Y}{\partial i}+\frac{\partial \mu}{\partial Z} \frac{\partial Z}{\partial i}+\frac{\partial \mu}{\partial \psi} \frac{\partial \psi}{\partial i}
$$$$
\frac{\partial \mu}{\partial X}=\operatorname{Re} \frac{\partial M}{\partial X}=\operatorname{Re}\left(\frac { 1 } { M D } \left\{2 X-1+j Z+\left(M^{2}-1\right)\right.\right.
$$$$
\left.\left.\left[1-j Z+\frac{2 Y^{2}(1-X-j Z) \cos ^{2} \psi}{S}\right]\right\}\right)
$$

$$
\begin{aligned}
\frac{\partial \mu}{\partial Y}= & \operatorname{Re} \frac{\partial M}{\partial Y}=\operatorname{Re}\left(\frac { ( M ^ { 2 } - 1 ) Y } { M D } \left\{\sin ^{2} \psi-\frac{1}{S}\left[Y^{2} \sin ^{4} \psi\right.\right.\right. \\
& \left.\left.\left.+2(1-X-j Z)^{2} \cos ^{2} \psi\right]\right\}\right)
\end{aligned}
$$

$$
\begin{aligned}
\frac{\partial \mu}{\partial Z}= & \operatorname{Re} \frac{\partial M}{\partial Z}=-\operatorname{Im}\left(\frac { 1 } { M D } \left\{X+\left(M^{2}-1\right)\right.\right. \\
& {\left.\left.\left[2-X-2 j Z+\frac{2 Y^{2}(1-X-j Z) \cos ^{2} \psi}{S}\right]\right\}\right) }
\end{aligned}
$$

where $I m=$ imaginary part of.

[Nоте: $\partial \psi / \partial i$ is calculated holding $\vec{\rho}$ constant and so measures the change in the direction of the earth's magnetic field in space.]

The quantities $\partial X / \partial i$ and $\partial Z / \partial i$ must be derived from the space variation of $X$ and $Z$ which are assumed. An arbitrary inhomogeneous ionosphere can be represented in this manner. If an earthcentered dipole field approximation is used for the magnetic field, then

$$
Y=Y_{e}\left(\frac{r_{0}}{r}\right)^{3} \sqrt{1+3 \cos ^{2} \theta}
$$

where $Y_{e}=Y$ at the equator at the surface of the earth $\approx\left(8.7 \cdot 10^{5}\right) / f$. The vector components of $Y$ in spherical coordinates are

$$
\begin{aligned}
Y_{r} & =\frac{Y}{\sqrt{1+(1 / 4) \tan ^{2} \theta}} & \frac{\partial Y}{\partial r} & =-\frac{3 Y}{r} \\
Y_{\theta} & =\frac{1}{2} Y_{r} \tan \theta & \frac{\partial Y}{\partial \theta} & =\frac{-3 Y \cos \theta \sin \theta}{1+3 \cos ^{2} \theta} \\
Y_{\phi} & =0 & \frac{\partial Y}{\partial \phi} & =0
\end{aligned}
$$

where

$$
\begin{aligned}
& \theta=\text { colatitude and } \\
& \phi=\text { longitude. }
\end{aligned}
$$

The derivatives of $\psi$ for a dipole magnetic field are

$$
\begin{aligned}
& \frac{\partial \psi}{\partial r}=0 \\
& \frac{\partial \psi}{\partial \theta}=\frac{\partial \psi}{\partial \gamma} \frac{\partial \gamma}{\partial \theta}
\end{aligned}
$$

where $\gamma=$ angle between $\vec{Y}$ and the radial (see fig. 1).

[Note: $\gamma=\tan ^{-1}(-1 / 2 \tan \theta)$ for the dipole field.]

$$
\begin{aligned}
& \frac{\partial \psi}{\partial \phi}=0 \\
& \frac{\partial \psi}{\partial \gamma}=\frac{\sigma_{\tau} Y_{\theta}-\sigma_{\theta} Y_{\tau}}{\sigma Y \sin \psi} \\
& \frac{\partial \gamma}{\partial \theta}=\frac{1}{2 \cos ^{2} \theta+(1 / 2) \sin ^{2} \theta} .
\end{aligned}
$$

[Note: When $\psi \rightarrow 0$, both numerator and denominator of $\partial \psi / \partial_{\gamma}$ go to zero and $\partial_{\psi} / \partial_{\gamma \rightarrow 1}$.] 


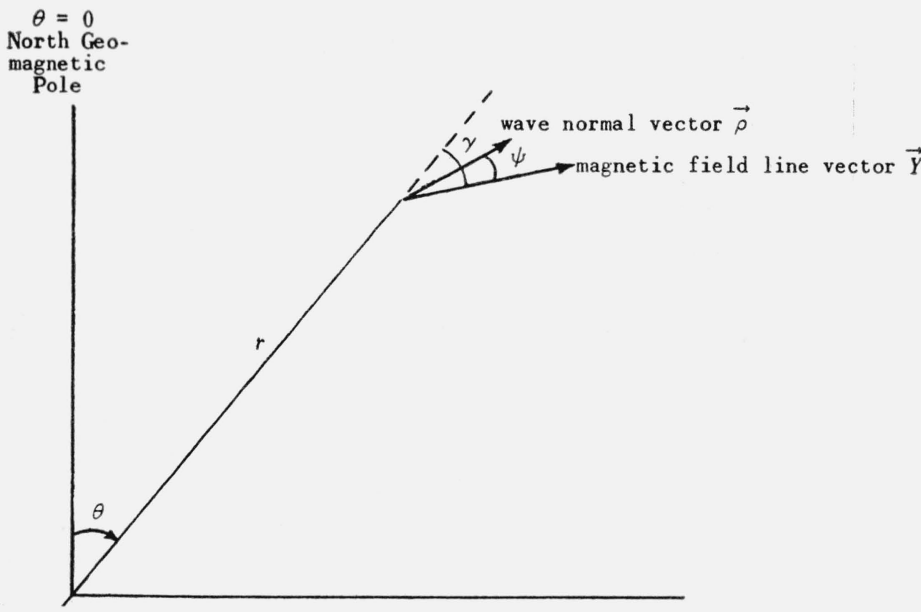

Figure 1. Coordinate system for two dimensions, $\mathrm{r}-\theta$.

It is usually desired to compute the following additional quantities along with the ray path:

(1) Path length, $S$

$\frac{d S}{d t}=\frac{1}{\mu^{2}} \sqrt{\mu^{2}+\left(\partial_{\mu} / \partial \psi\right)^{2}}=\frac{1}{\text { ray refractive index }}=\frac{1}{\mu \cos \alpha}$.

(2) Time of travel, $T$, also called group delay

$\frac{d T}{d t}=\frac{1}{c}\left(1+\frac{f}{\mu} \frac{\partial \mu}{\partial f}\right)$

$\frac{\partial \mu}{\partial f}=\operatorname{Re} \frac{\partial M}{\partial f}=\operatorname{Re}\left(\frac{1}{M D f}\left\{X(2 X+j Z)+\left(M^{2}-1\right)\right.\right.$

$\left[2-2 j Z-j Z X+\frac{2}{S} Y^{2} \cos ^{2} \psi\right.$

$(1-X-j Z)(1+X)]\})$

(3) Absorption, $A$, in nepers

$$
\frac{d A}{d t}=-\frac{\omega}{c} \frac{K}{\mu} ; \quad \omega=2 \pi f
$$

\subsection{Notes on the Method of Digital Computation Used}

\section{a. Initial Conditions Needed}

Initial values for $r, \theta, \phi, \rho_{r}, \rho_{\theta}, \rho_{\phi}$ are needed. To get $\rho_{r}, \rho_{\theta}$, and $\rho_{\phi}$, the wave normal direction can be inserted as an initial condition. $\mu$ is then calculated and the $\rho$ 's obtained. For example, let $x_{i}=\left(\rho_{i} / \rho\right)=$ direction cosines of wave normal with respect to the local coordinate axes. Then, since $\rho=\mu, \rho_{i}=\mu x_{i}$.

\section{b. Stability Condition}

The six basic ray differential equations contain one more degree of freedom than there are constraints explicitly expressed. That is, it only takes 5 quantities to define a vector field (three space coordinates and two direction cosines). The sixth equation is implicit in the definition of $|\vec{\rho}|=\mu$. The round-off errors involved in calculating $\mu$ and in integrating $d \rho_{i} / d t$ will result in $|\vec{\rho}| \neq \mu$ after a few steps of integration unless an additional constraint is imposed. Thus, to maintain the equality, we correct the $\rho_{i}$ 's at each point in the manner

$$
\rho_{i \text { corrected }}=\rho_{i} \stackrel{\mu}{\rho} \text {. }
$$

This correction is made as early as possible in the derivative calculation, i.e., immediately after calculation of $M$. Since the magnitude of $\vec{\rho}$ is not used in the calculation of $M$ (only the wave normal direction is used), no errors arising from $|\vec{\rho}| \neq \mu$ are allowed to accumulate by this procedure.

$$
\text { c. Interval Size }
$$

The $\Delta t$ to use depends upon the accuracy required, the frequency, the size of refractive index gradients encountered along the ray path, and the stability and accuracy of the numerical method of solution of the differential equations used. For the whistler ray paths, shown later in this report, the interval size was changed when the local truncation error became either too large or too small. The local truncation error was measured by noting the difference between the predicted values for the next point and the values obtained by application of the first corrector (an Adam's predictor-corrector method of solution of the D.E. equations was used). When this difference became less than $5 \cdot 10^{-7}$, the interval size was doubled using an extrapolation formula in a special subroutine. When the difference became greater than $10^{-4}$, the interval size was halved. The corrector was applied only once every 5 or 10 points and only a predictor was used for the in-between points. This was done because application of a corrector and subsequent doubling or halving costs several "points" worth of time. Thus it is undesirable to do this very often. The interval size used for these whistler calculations was approximately one-half an earth radius. Calculation was started at the top of the $F_{2}$ layer. 


\section{d. Other Comments}

The bulk of computation time is spent in calculating the derivatives of $M, \mu$, etc. Only a small fraction of the total time is actually spent in the numerical method of solution calculations. Therefore, a method of solution should be chosen that minimizes the number of times the derivatives have to be calculated, and that makes maximum use of the derivatives already calculated. Thus the RungeKutta methods are long and cumbersome for these equations. A predictor-corrector method with a minimum number of corrector cycles applied seems to be about the best. Perhaps even a high-order predictor (5th or 6 th order) might be best, since this would increase the interval size one could use without increasing the calculation time proportionately.

The main weakness of the program used for these calculations is the instability of the extrapolation formula used for doubling. This formula has to jump ahead two intervals. It would be better to carry more points along with the calculations and use a higher-order extrapolation formula that only jumps ahead one space or better still, carry sufficient points to use no extrapolation formula at all.

If these equations are to be used for frequencies high enough that the earth's magnetic field can be ignored (frequencies much higher than the local gyrofrequency) then a separate routine should be written for this case, since the equations simplify to a much easier and faster computation. If the same routine is used, many of the expressions for the derivatives become indeterminate when $Y=0$. Most of these derivatives actually become zero, but special tests would have to be installed in the program to handle this case.

\subsection{Computations}

\section{a. General Procedure}

The differential equations were programed for numerical solution on an Electrodata 205 computer. Only the two-dimensional case of propagation in the magnetic meridian was utilized in these computations. Also, no losses were included $(\nu=0)$. The calculation of a whistler path was begun with the assumed initial conditions at an altitude of $300 \mathrm{~km}$. This altitude corresponds roughly with the maximum ionization level of the $F_{2}$ layer.

An electron density profile was assumed for altitudes greater than this.

The computations are organized into the following categories according to the electron density profile used and the parametric variation examined:

(1) Exponential model-variation of frequency,

(2) Exponential model-variation of initial latitude,

(3) Exponential model-variation of initial wave normal direction,

(4) Constant density model, and

(5) Miscellaneous.

The exponential model used in all of the first three categories is:

$$
N=180,000 \exp [-4.183119(r-1.0471)]
$$

where

$N=$ electron density in electrons per cubic centimeter. $r=$ radial distance from center of earth in earth radii.

$(r=1.0471=300 \mathrm{~km}$ above the surface of the earth at the beginning of the path computation).

These particular values of electron density and scale height were obtained from Maeda and Kimura [1956].

\section{b. Exponential Model-Variation With Frequency}

Figure 2(a) gives the results of ray path computations at the frequencies $5,10,20,25,30,50,100$, $200,400,1000 \mathrm{kc} / \mathrm{s}$. Figures 2 (a) and (b) show the ray path for each of these frequencies. The initial wave normal direction for each of these paths is vertical (wave normal angle $=0^{\circ}$ ). In order to exhibit the variation of certain variables in these computations, figures 2 (c) to 2 (i) have been plotted.

Some of the interesting features of these computations may be listed as follows:

\section{c. Exponential Model-Variation With Latitude}

Figure 3 gives the results of ray path computations initiating at north latitudes of $10,20,30,35,40,45$, 50 , and $60^{\circ}$. Figures 3 (a) to (i) show the ray paths for each of these latitudes as well as the variation of wave normal direction over the path. Additional data from these computations have been plotted in figures 3 (j) to (o).

\section{d. Exponential Model-Initial Wave Normal Variation}

Figure 4 gives the results of ray path computations with initial wave normal angles $10,5,-5$, and $-10^{\circ}$ measured positively clockwise from vertical (along the radial). Figures 4 (a) to (d) show the ray paths and wave normal direction over these paths. The wave frequency is $10 \mathrm{kc} / \mathrm{s}$. Additional data from these computations have been plotted in figures 4 (e) to $(\mathrm{g})$.

The behavior of the wave normal over the path changes only negligibly with these changes in initial wave normal direction.

\section{e. Constant Density Model}

Figure 5 gives the results of ray path computations with constant electron density. The characteristics of a constant density model are as follows. Since there is no electron density gradient, the total refractive index gradient is formed by the magnetic field. Also, since the refractive index gradient determines the change in wave normal which in turn determines the bending of the ray path, the bending of the ray path is determined only by the guiding effect of the magnetic field, not by the electron density. Thus the ray path is the same regardless of the magnitude of the electron density. 


\section{f. Miscellaneous Computations}

Comparisons with Maeda's computations-Maeda (1951) used a very approximate method of numerical integration as well as an approximation to the refractive index gradient which is only very roughly correct. In order to compare his computations with the more exact formulation of Haselgrove, one of Maeda's published ray paths was compared with a similar computation using the present program in figure 6 . It can be seen that the difference is appreciable. The lack of comparison is not due to the quasi-longitudinal approximation of the refractive index function which was used by Maeda since this approximation was used also in Haselgrove's formulation for this particular computation. The difference between the Q. L. approximation and the full expression is too small to be shown. The differences between the two computed paths may be due to the relatively crude method of integration used by Maeda to numerically solve the differential equations.

Field-alined column model - In a recent theoretical paper it has been shown that if the ionization is field-alined it can act like a waveguide in trapping whistler energy [Smith, Helliwell, and Yabroff, 1960]. This would explain some of the very distinct and clear whistlers which have been recorded. In order to answer certain questions regarding the effects of field-alinement, the following models have been computed:

$$
\begin{aligned}
N=180,000\left\{1+C \exp \left[\frac{\left(b-b_{0}\right)^{2}}{2 D^{2}}\right]\right\} & \\
& \quad \exp [-4.183119(r-1.0471)]
\end{aligned}
$$

where

$b=r / \sin ^{2} \theta$ (the equation of a field line is $b=$ constant),

$b_{0}=2.094$ (this is a field line corresponding to 300 $\mathrm{km}$ altitude at a latitude of $45^{\circ}$ and is the center of the column),

$C=$ the modulation factor, i.e., the relative increase of maximum ionization at the center of the column over that of the surrounding background level of ionization, and

$D=$ the standard deviation of the column. (The column is of gaussian electron density distribution with the standard deviation measured in terms of the local distance between the field lines.) This makes more intuitive physical sense than making the thickness of the column constant at all points on the field line, since the individual electrons will tend to diffuse easily along the lines of force but not across them.

For these computations $D=0.029$ which corresponds to a standard deviation of about $35 \mathrm{~km}$ at an altitude of $300 \mathrm{~km}$, and about $215 \mathrm{~km}$ at the top of the path.

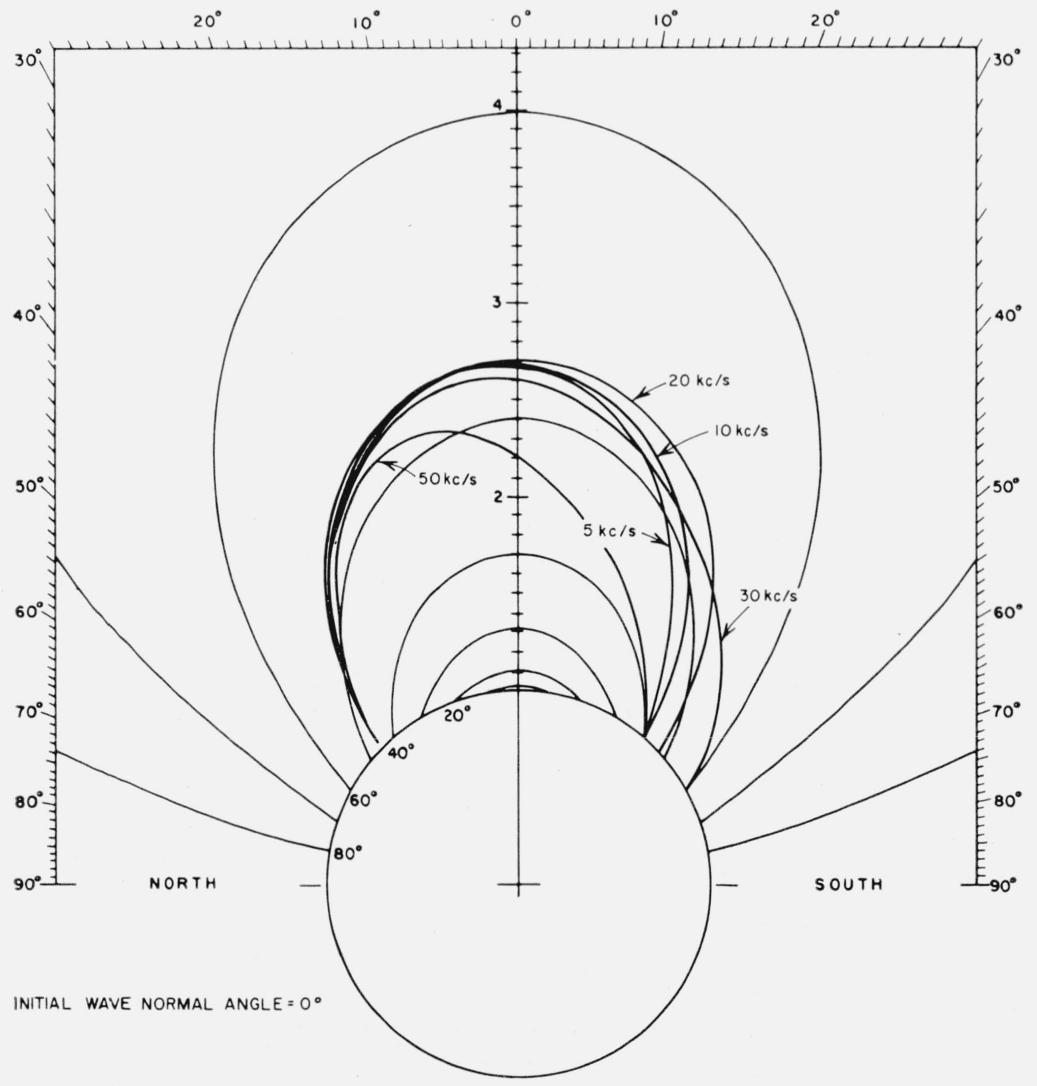

Figure 2(a). Ray paths at various frequencies. 


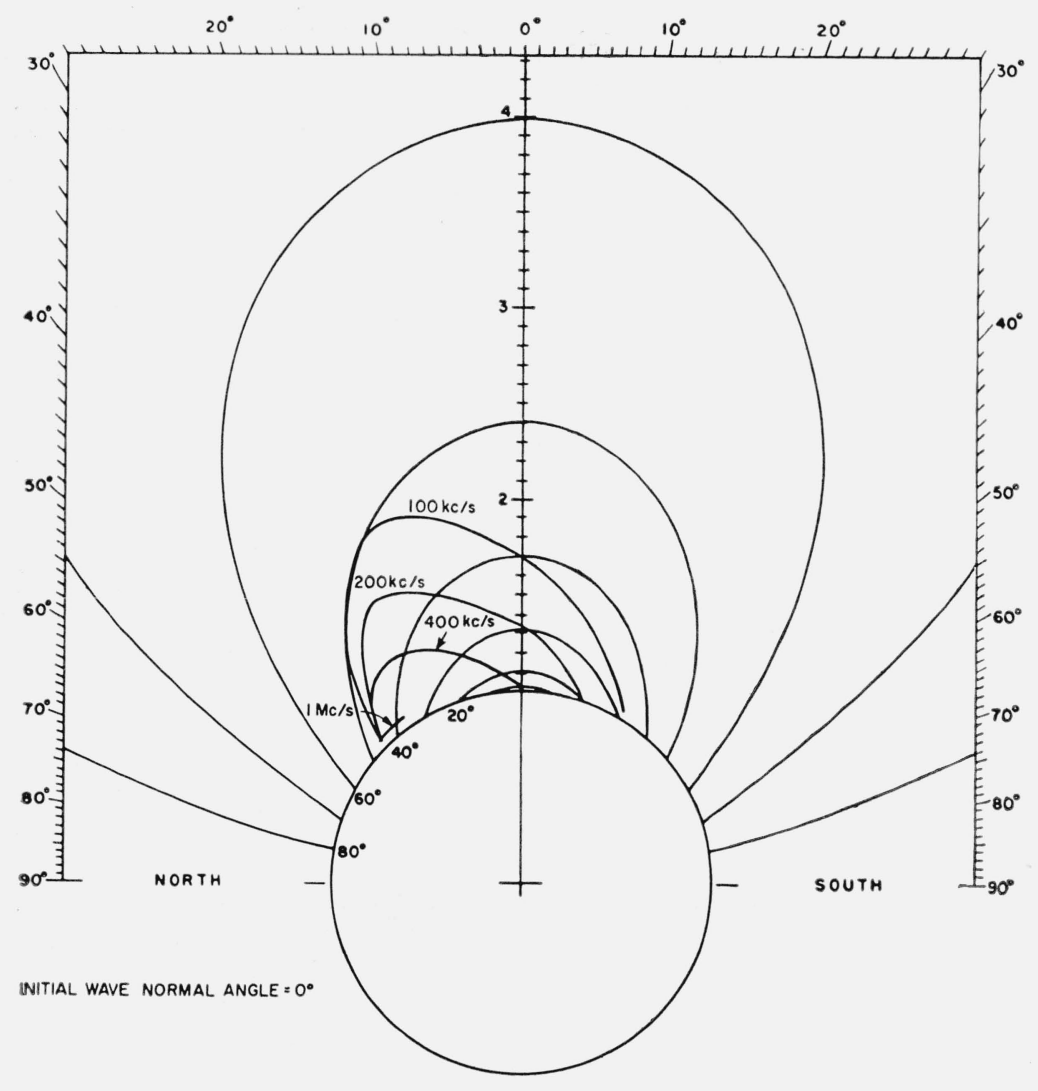

Figure 2(b). Ray paths at various frequencies.

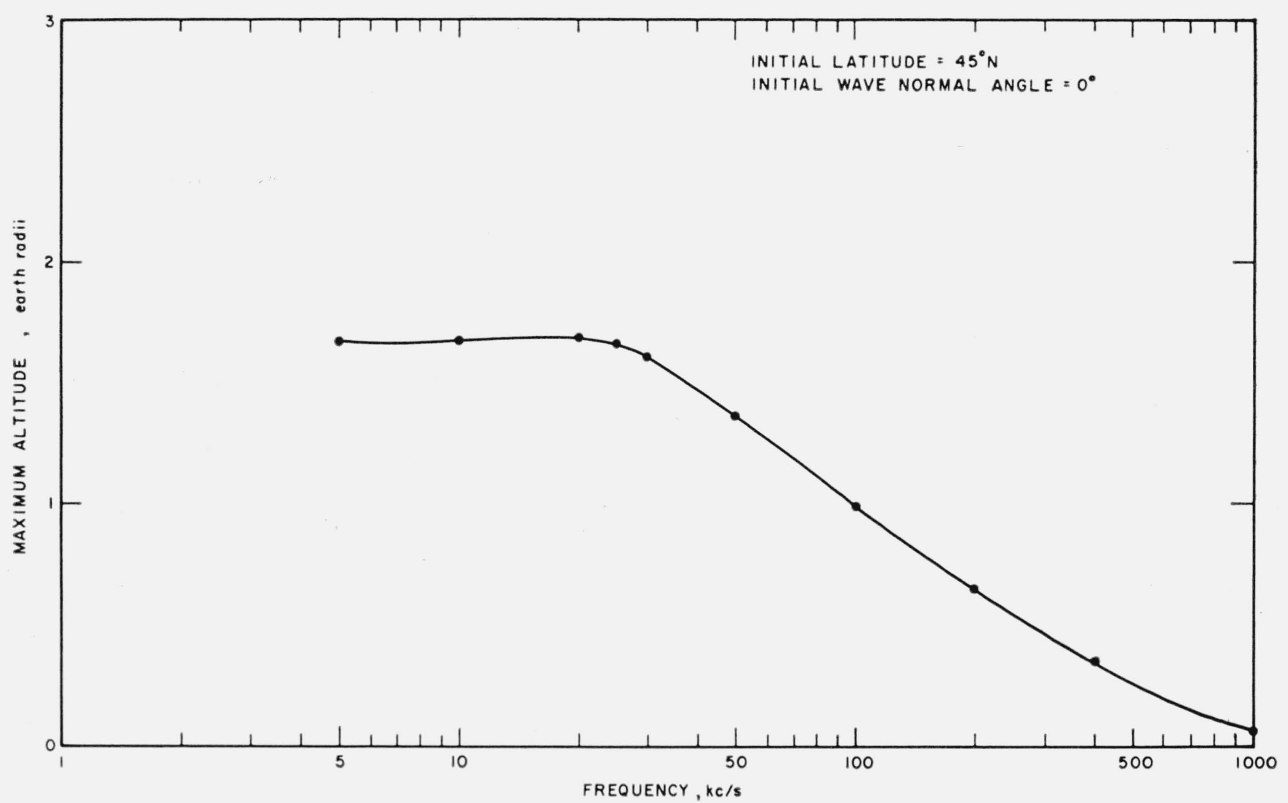

Figure 2(c). Maximum altitude versus frequency.

The maximum height of the path remains essentially constant up to the frequency of minimum time 'delay [the nose frequency, which is seen to be at about $20 \mathrm{kc} / \mathrm{s}$ in fig. $2(\mathrm{~d})$ ] at which point the maximum path height begins decreasing. 


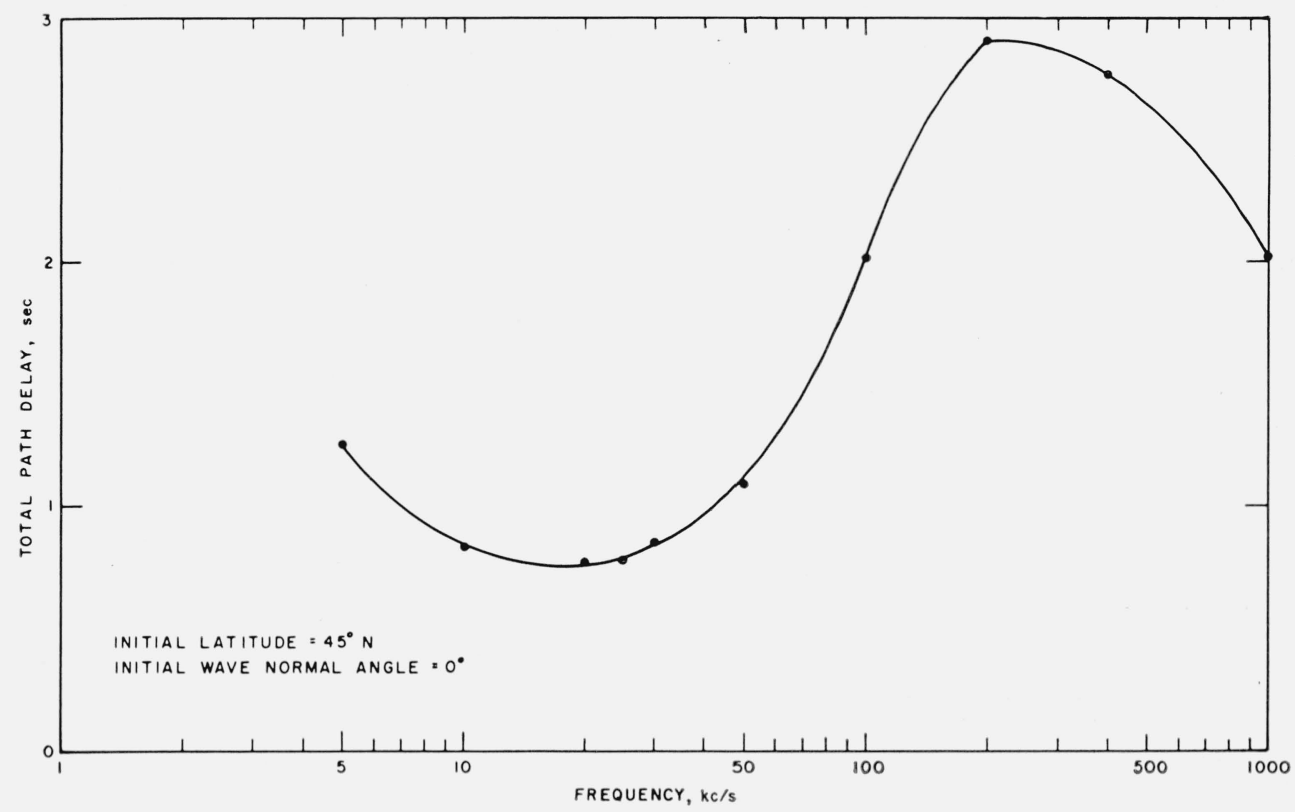

FiguRE 2(d). Total path delay versus frequency.

The time delay decreases in the expected "Eckersley" fashion until the nose repion is reached. This region corresponds ito the frequency reaching approximately 45 percent of the gyrofrequency at the top of the path. These calculations confirm this behavior. The time delay at higher frequencies increases until the effect of the shortening of the path length at high frequencies overbalances the decrease in group velocity to cause the time delay to begin to decrease again above $200 \mathrm{kc} / \mathrm{s}$.

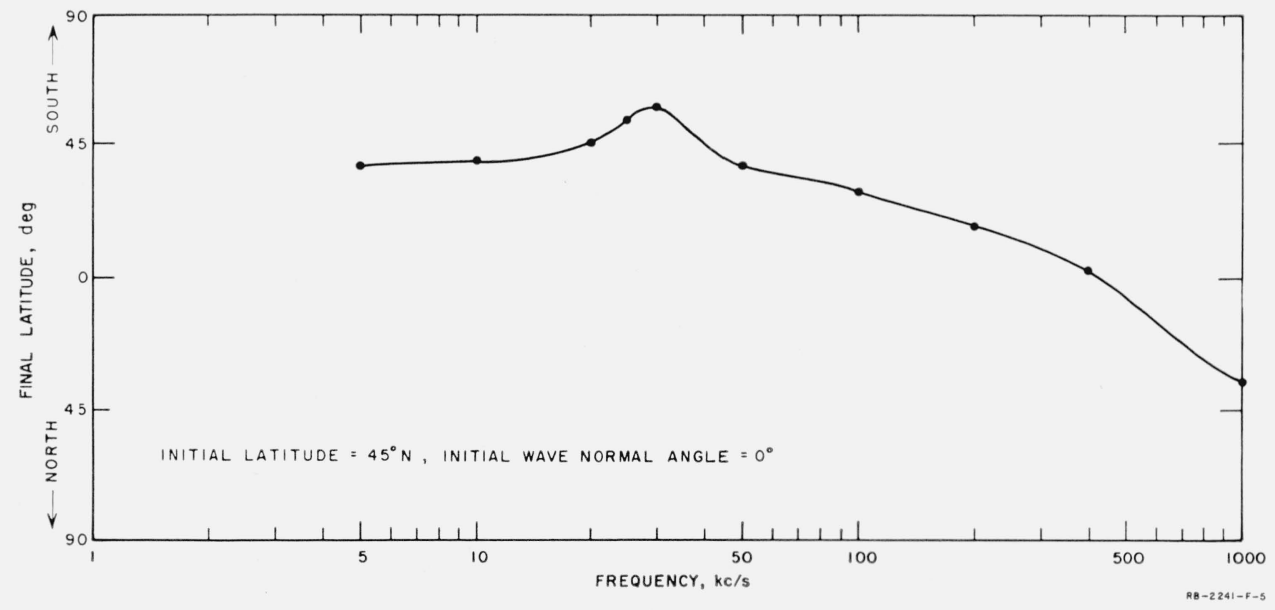

Figure 2(e). Final latitude versus frequency.

The final latitude remains fairly constant at frequencies up to the nose frequency. This suggests some latitude focusing in the "Eckersley" region. A bove the nose frequency, the final latitude starts shifting toward the pole until the effect of the shortening path length at higher frequencies overcomes this tendency and results in a shift back toward the equator. 


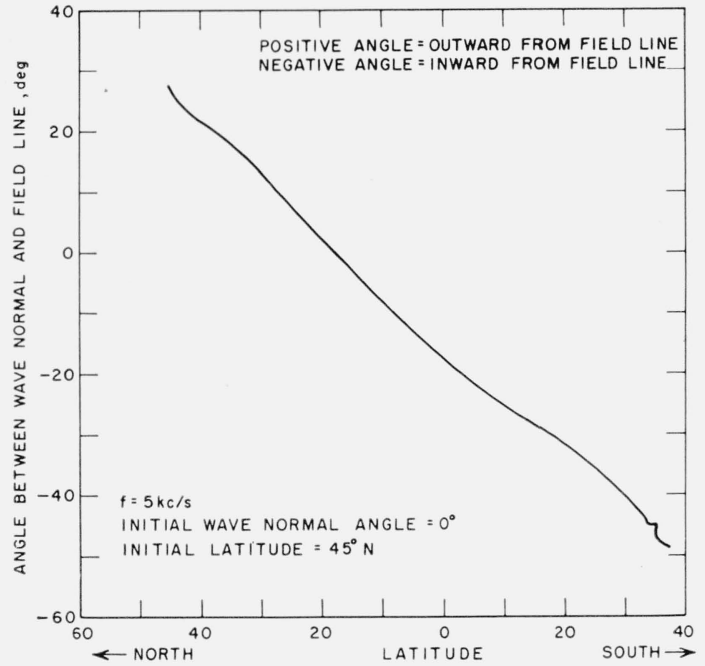

Figure 2(f). Wave normal-field line angle along the path.

At frequencies below the nose frequency, the wave normal swings steadily below the field line as the path progresses. As the nose frequency is approached, the wave normal tends to oscillate about a position approximately ten degrees above the field line. At higher frequencies, the wave normal swings outward after an initial dip toward the field line.

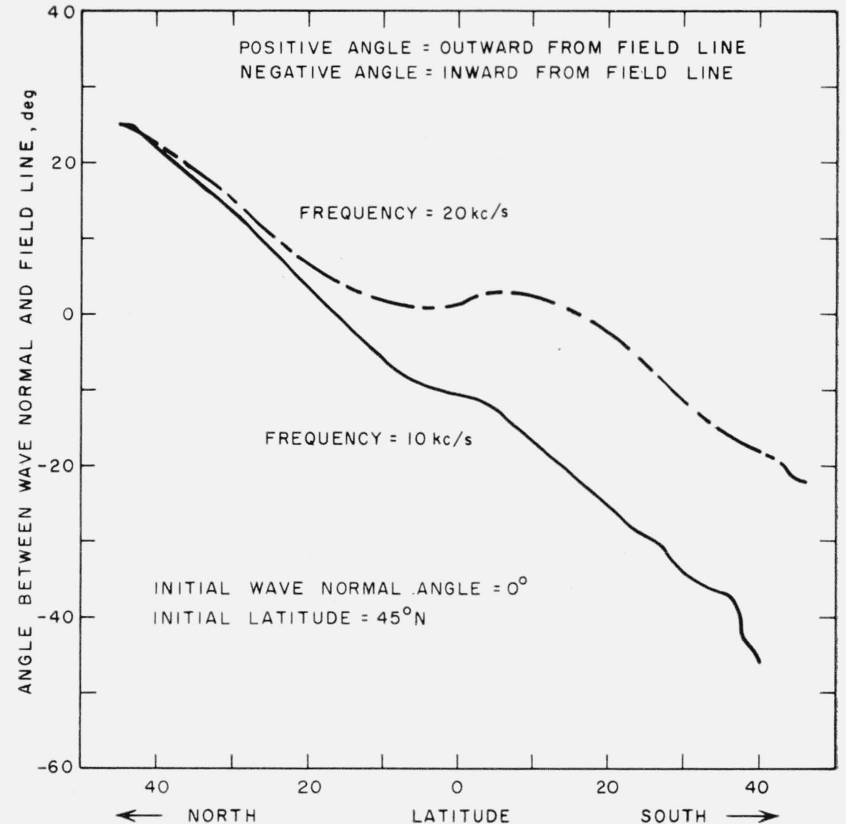

Figure 2(g). Wave normal-field line angle along the path.

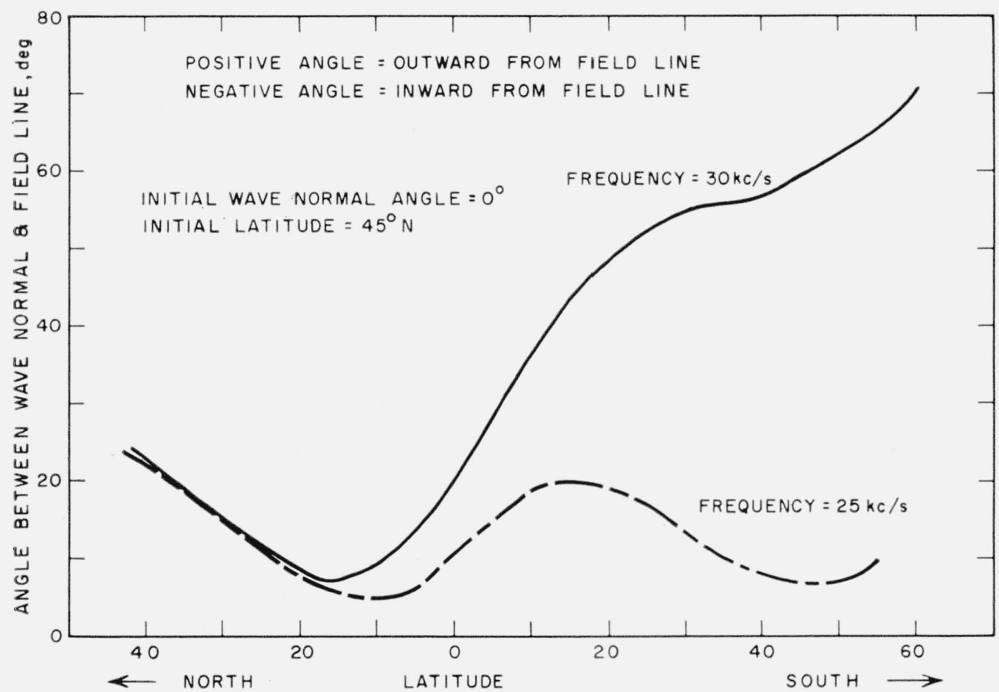

Figure 2(h). Wave normal-field line angle along the path.

In the first two computations shown in figures 7 and 8 the initial wave normal direction is vertical. When the modulation is 0.2 , good guiding occurs. When the modulation is reduced to 0.1 , the ray is not guided because the initial wave normal direction is far from the field-line direction and the ray gets pulled out of the column before the wave normal gets properly lined up with the field line. This is in agreement with theoretical predictions [Smith, Helliwell, and Yabroff, 1960]. In the third case shown in figure 9 , the wave normal was set along the field line. In that case, the guiding is even better than for the higher modulation of case 1 . The final case shown in figure 10 shows the modulation reduced to zero with the initial wave normal direction on the field line. The guiding of this ray is still good, but less so than for the previous case of 0.1 modulation.

These calculations support the theoretical predictions [Smith, Helliwell, and Yabroff, 1960] that wave components whose wave normals lie within a certain angle with respect to the direction of the magnetic field will be trapped. 


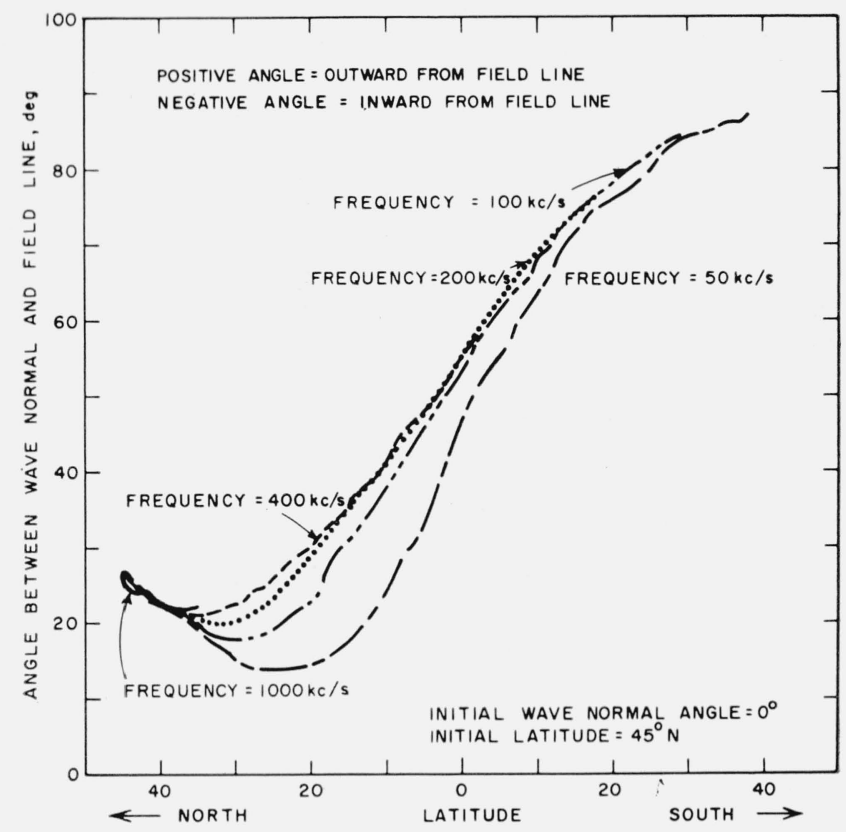

Figure 2(i). Wave normal-field line angle c.long the path.

Figure 3(a). Ray path initiating at $10^{\circ} \mathrm{N}$ latitude.

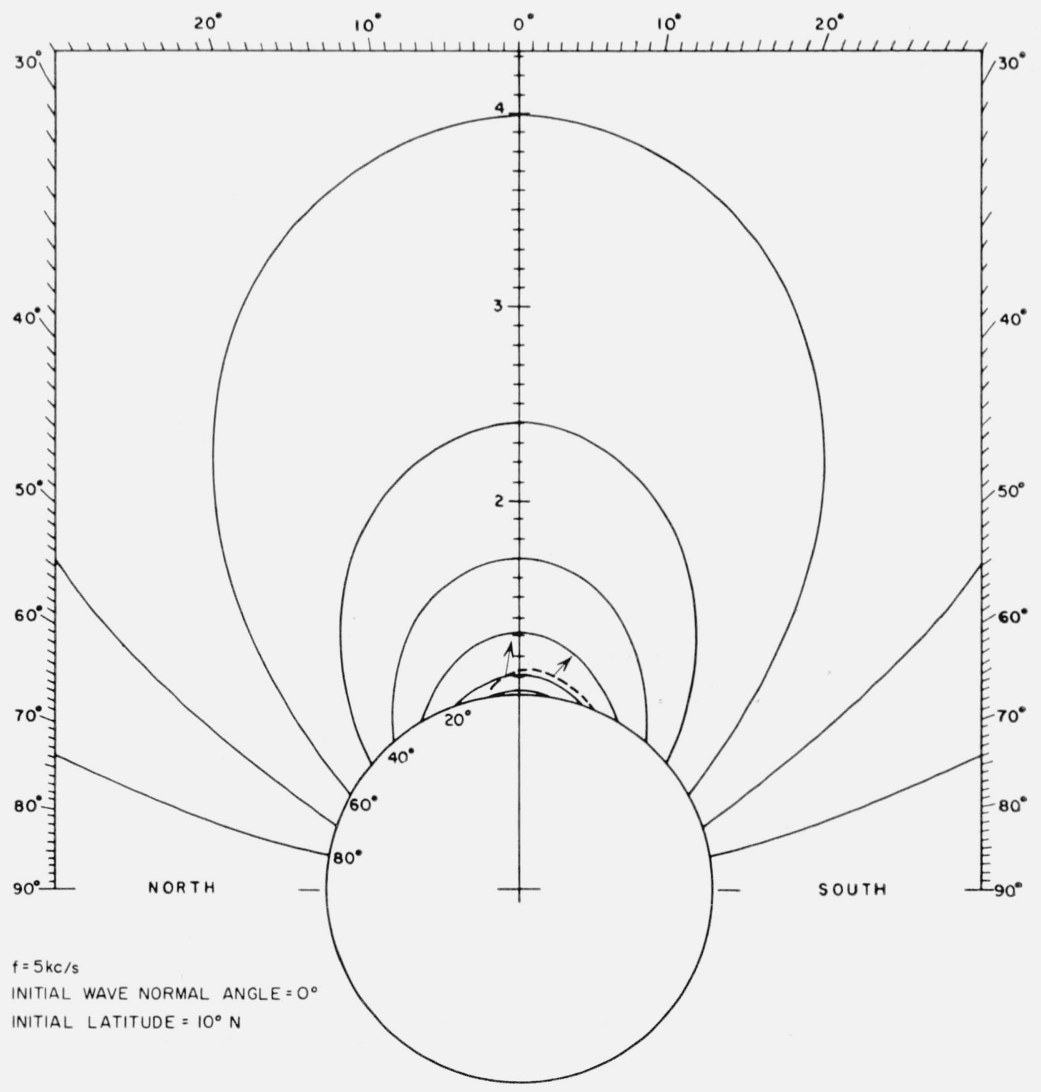




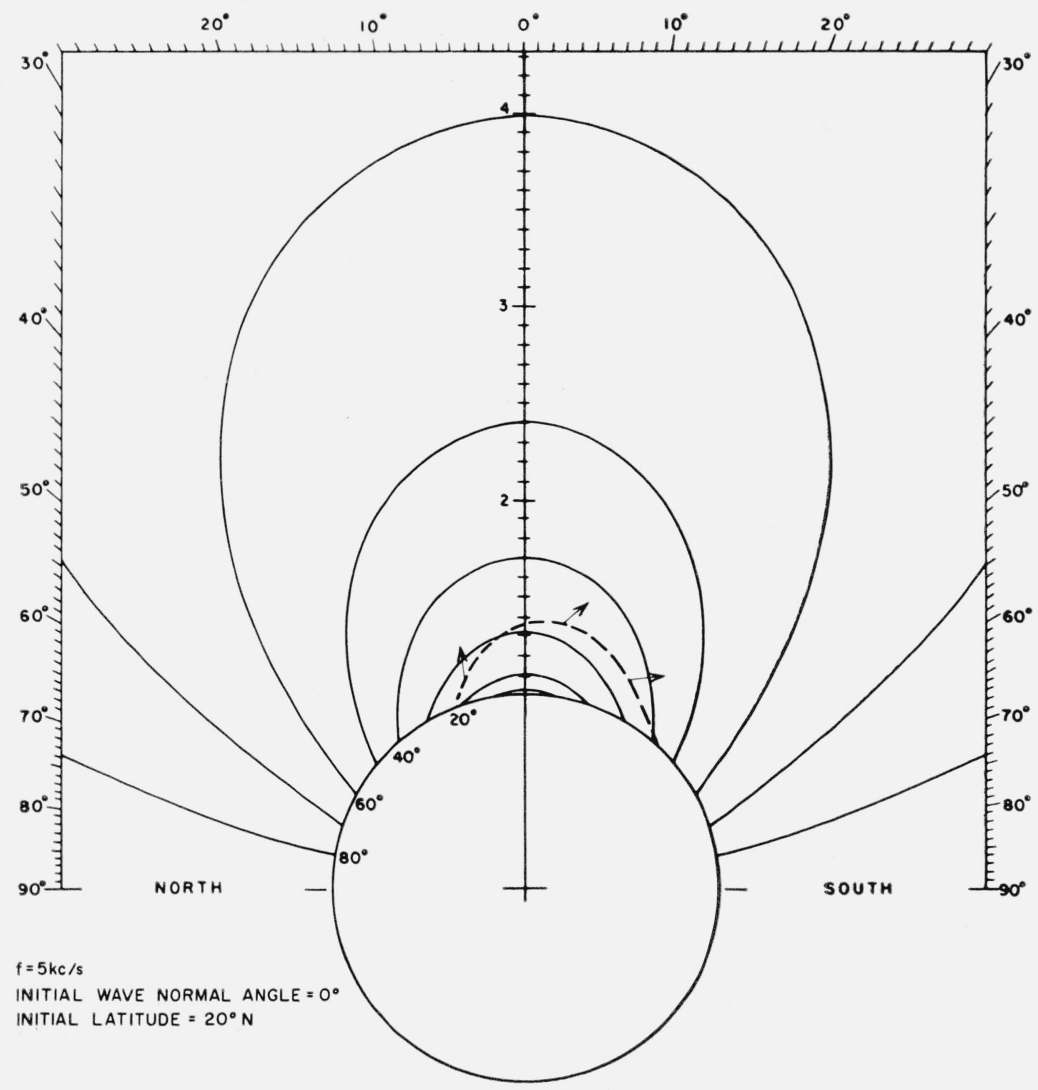

Figure 3(b). Ray path initiating at $20^{\circ} \mathrm{N}$ latitude.

Figure 3(c). Ray path initiating at $30^{\circ} \mathrm{N}$ latitude.

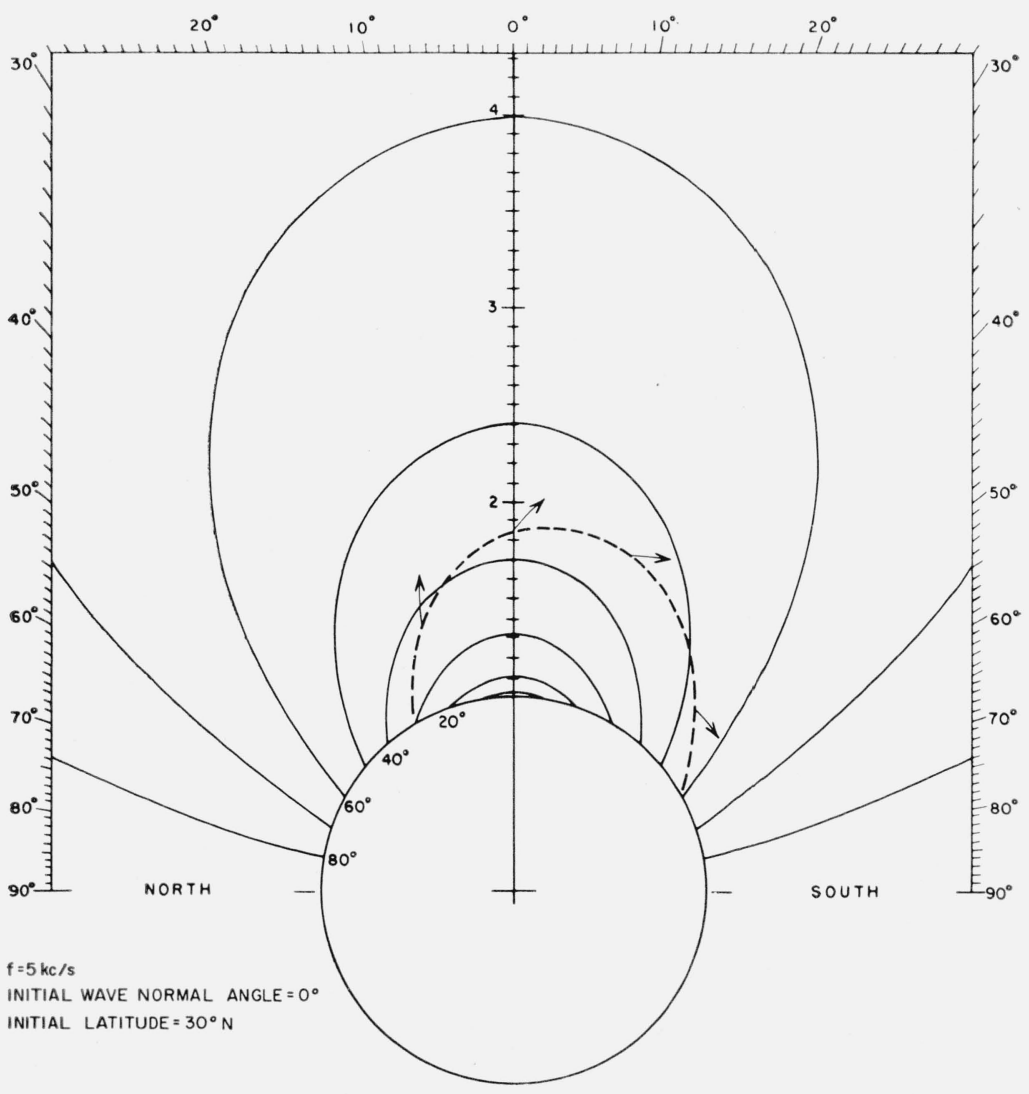




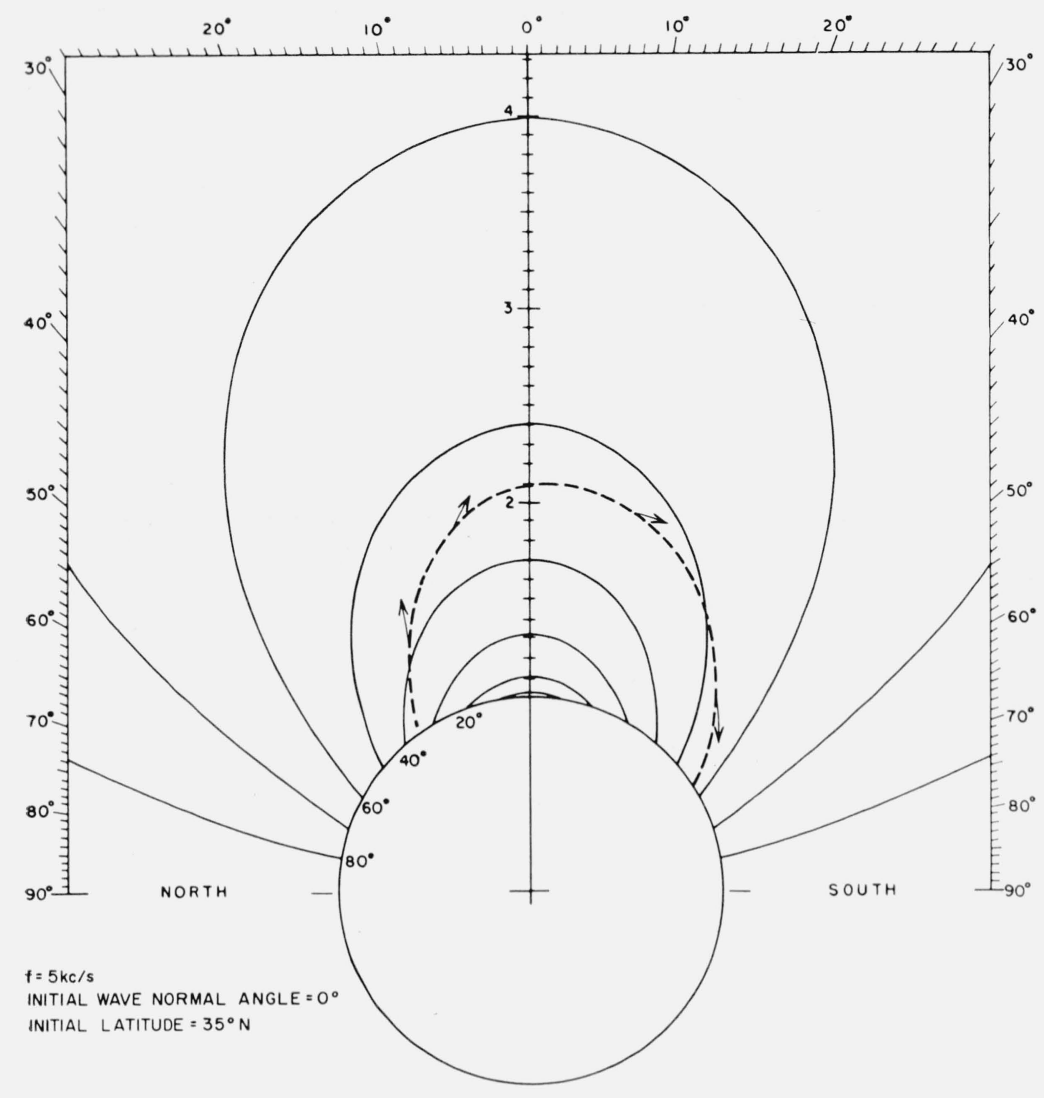

Figure 3(d). Ray path initiating at $35^{\circ} \mathrm{N}$ latitude.

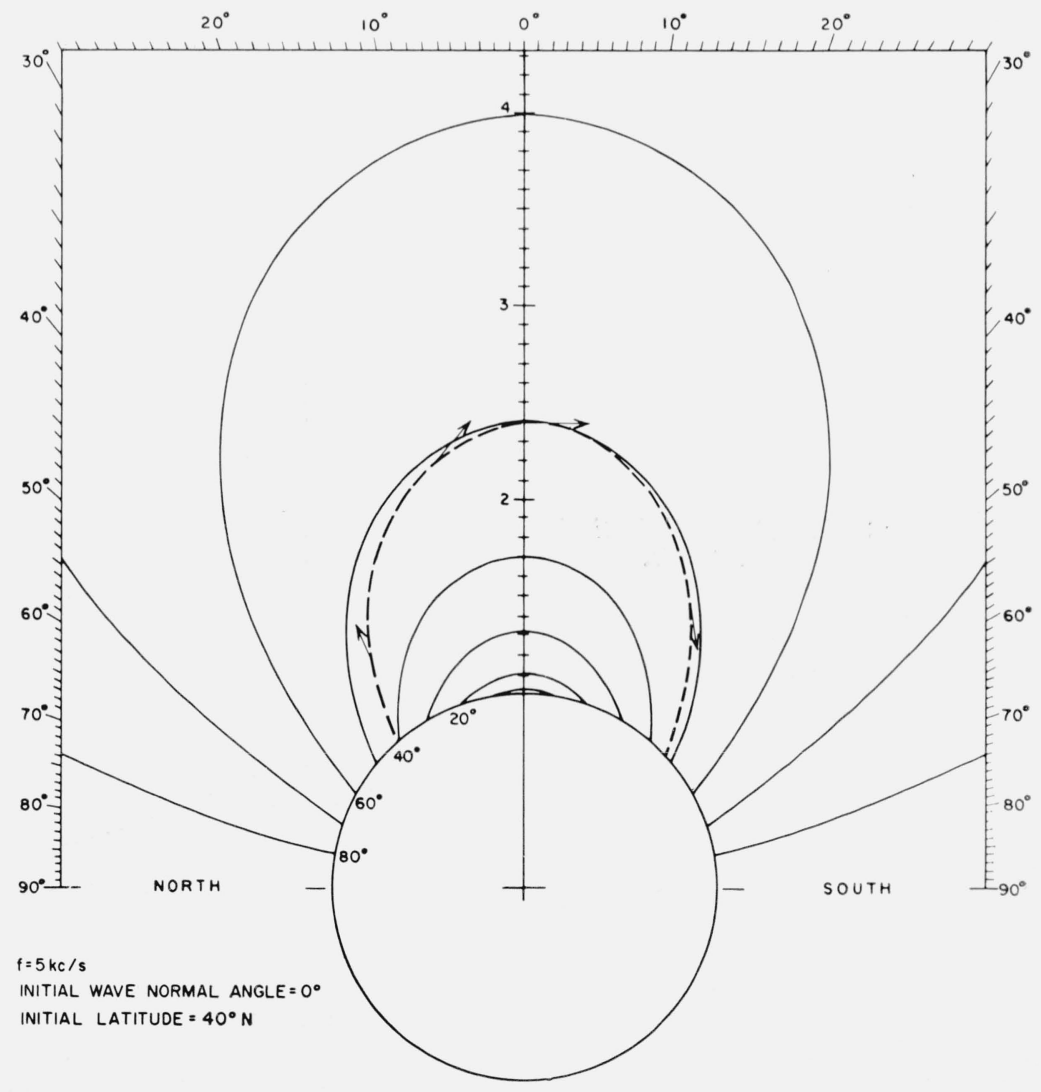




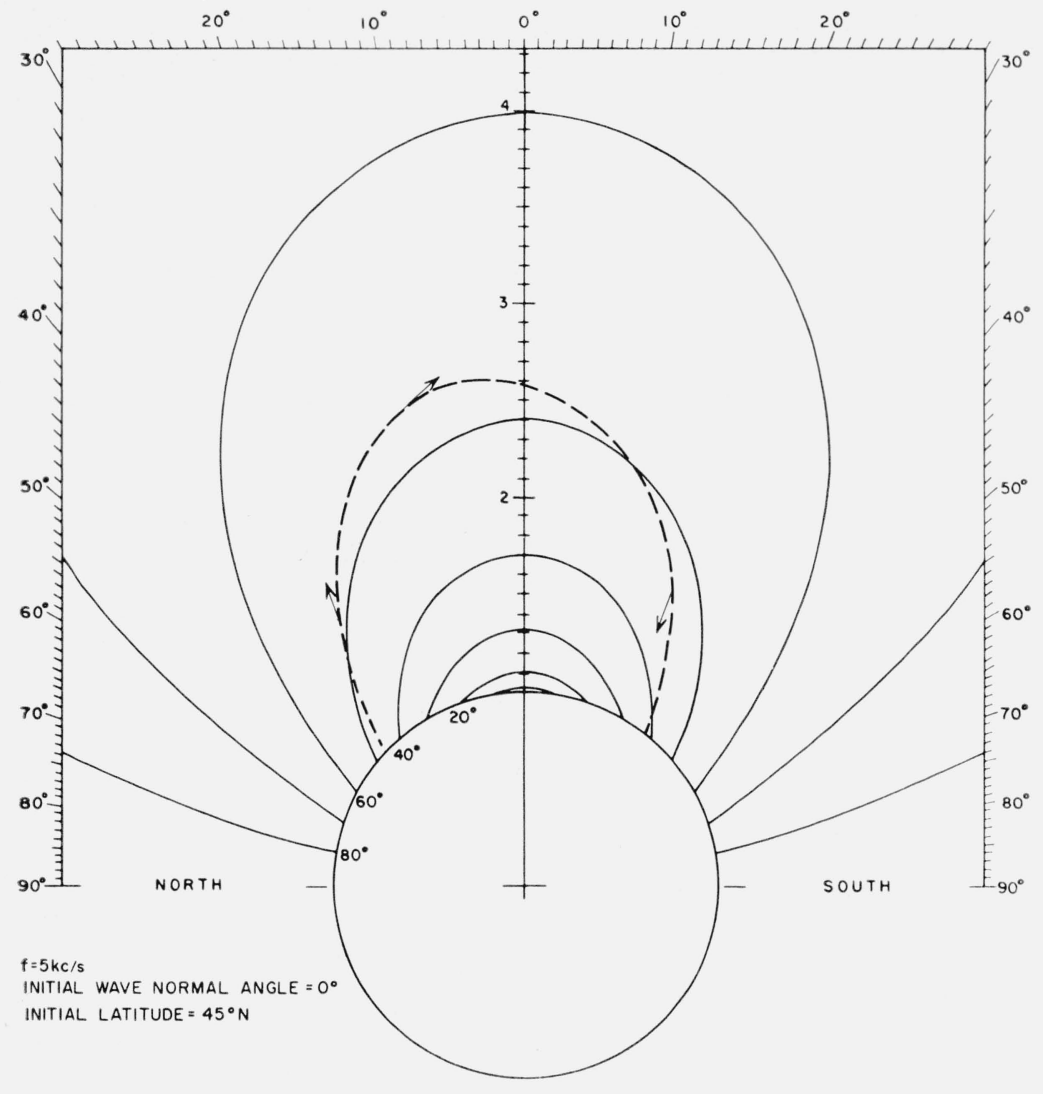

Figure 3(f). Ray path initiating at $45^{\circ} \mathrm{N}$

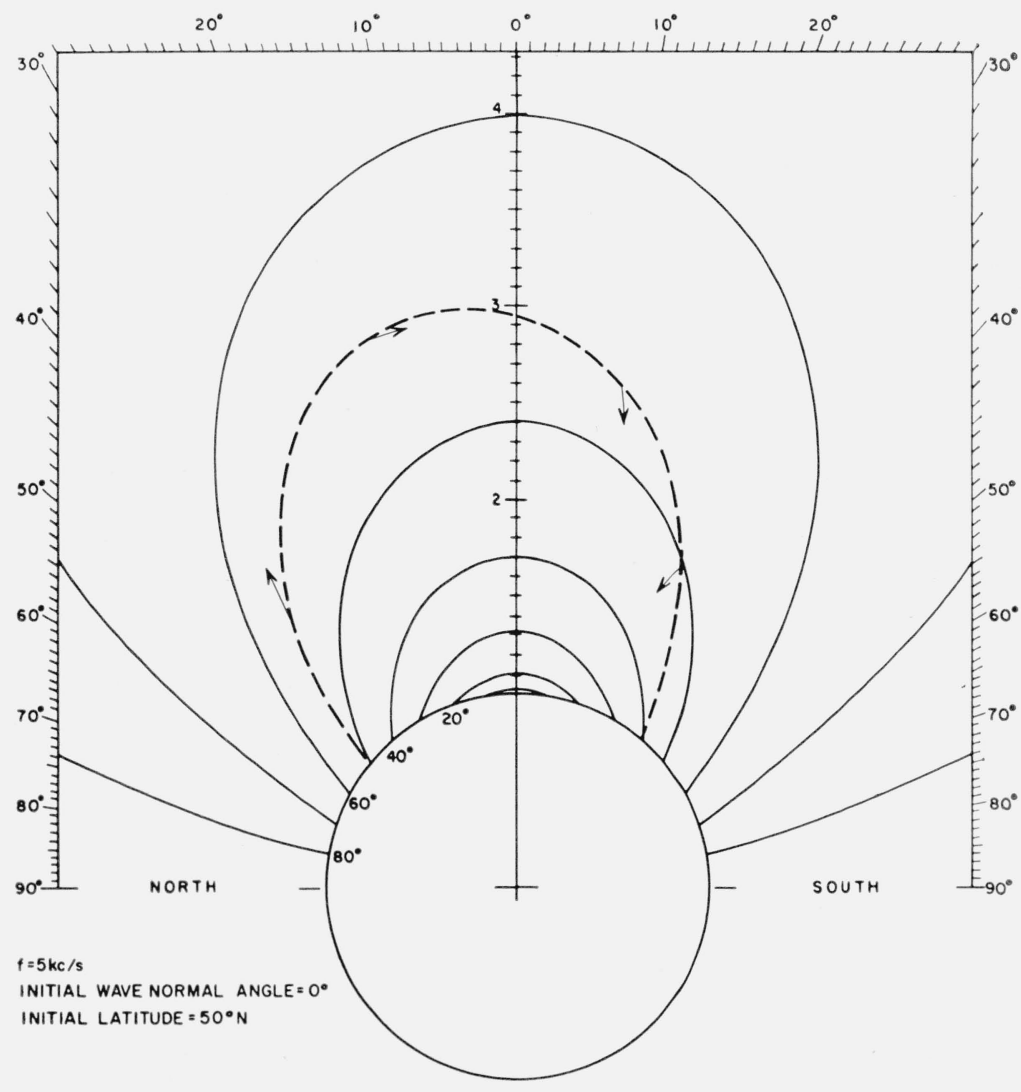

Figure $3(\mathrm{~g}) . \quad$ Ray path initiating at $50^{\circ} \mathrm{N}$ latitude. 


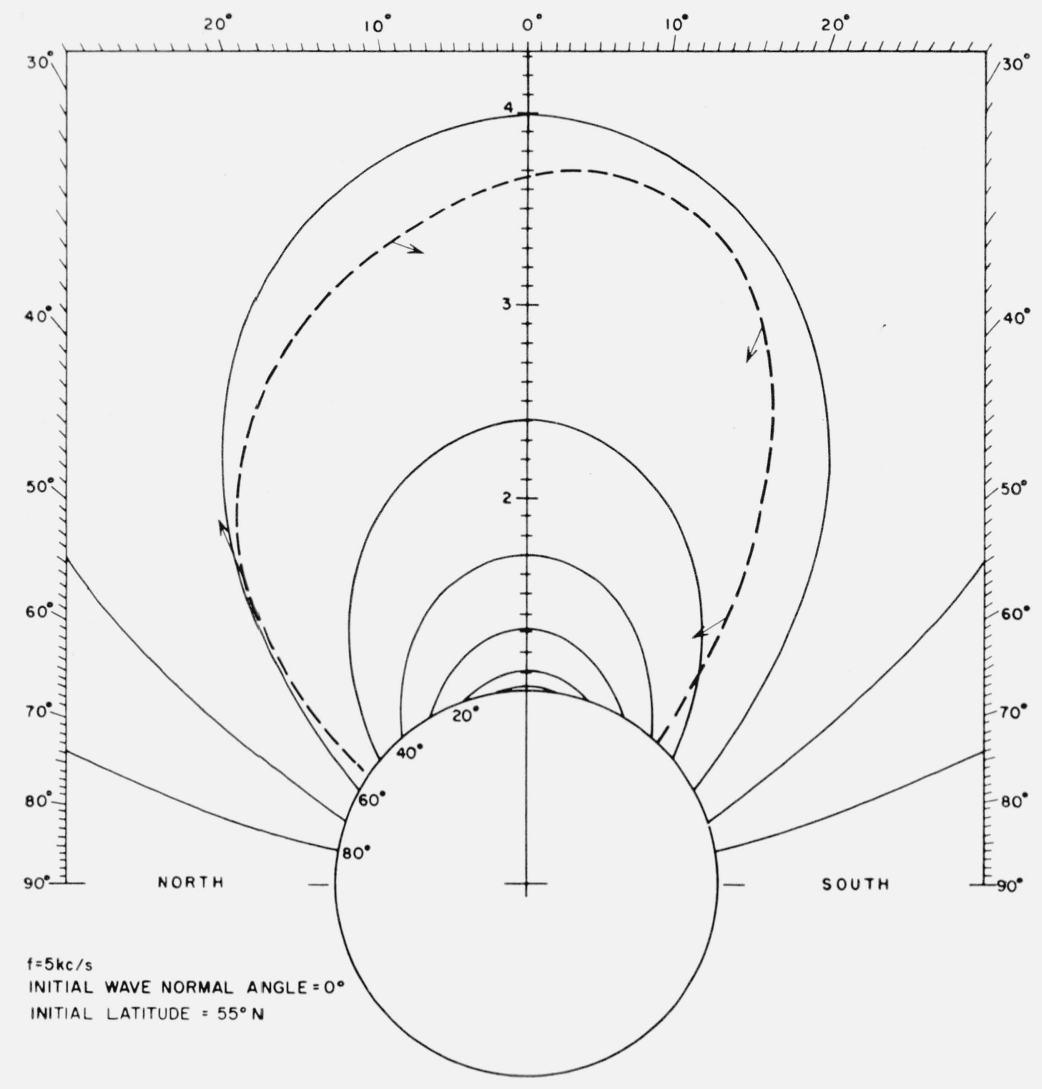

Figure 3(h). Ray path initiating at $55^{\circ} \mathrm{N}$ latitude.

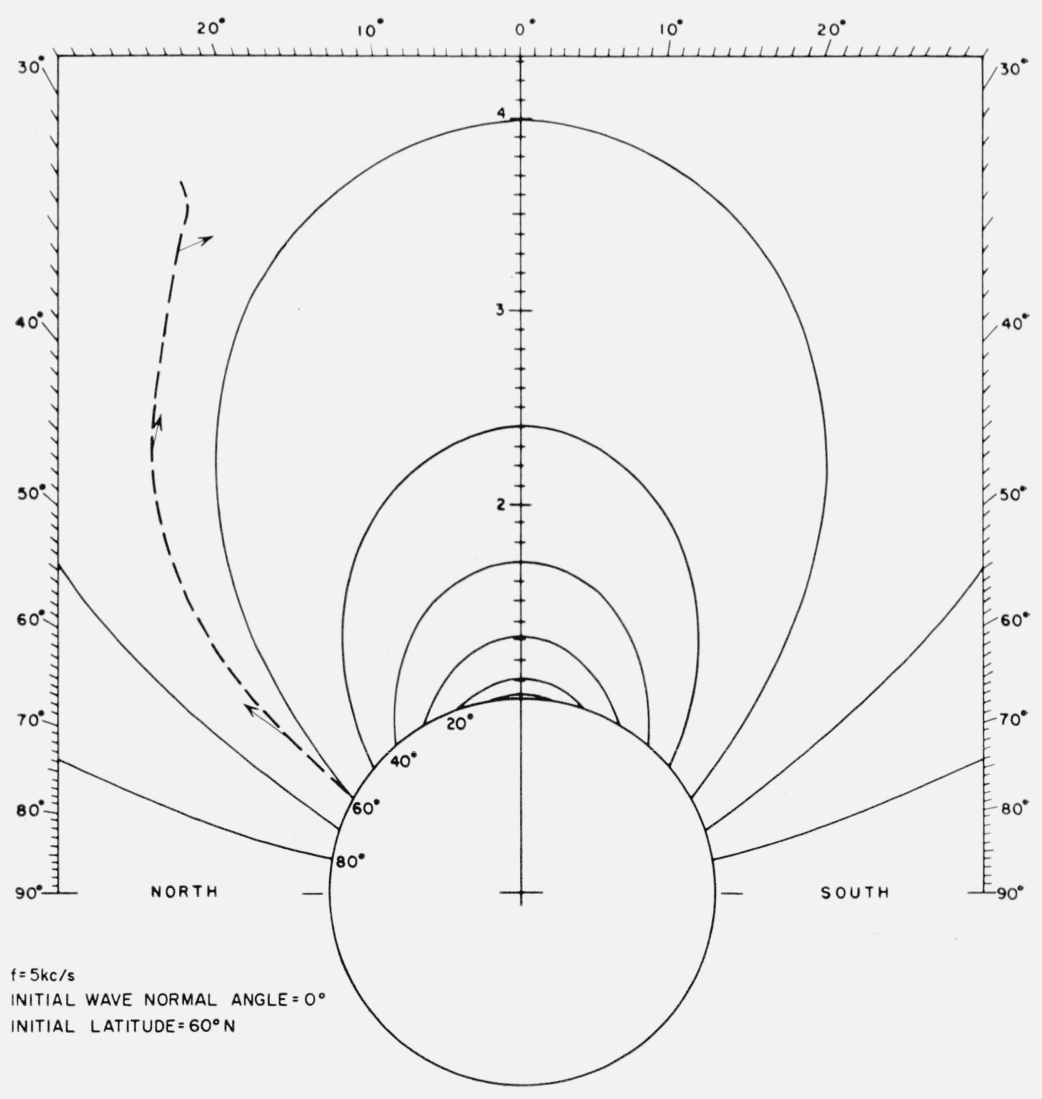




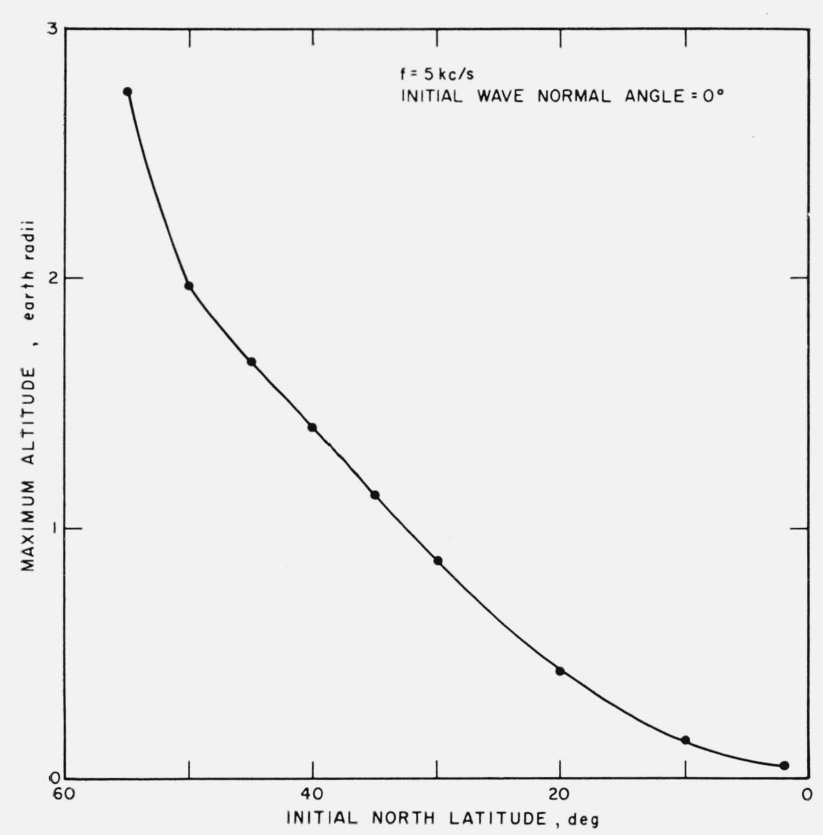

FIGURE 3(j). Maximum altitude versus initial latitude.

The maximum height of the path is seen to increase very rapidly with increasing initial latitude until an altitude is reached at which the plasma frequency becomes equal to the wave frequency. This represents the usual reflection level.

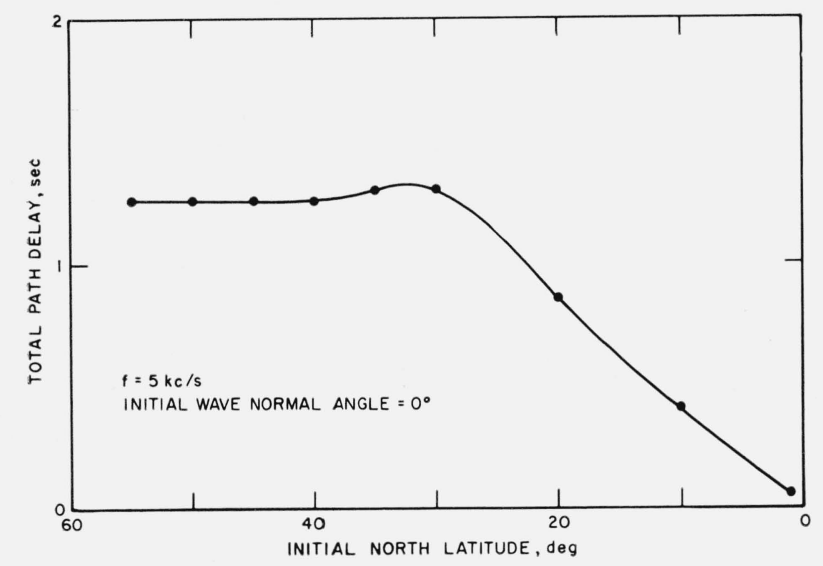

Figure 3(k). Total path delay versus initial latitude.

The total time delay is seen to be fairly constant over a wide range of initial latitude. This may be a bit surprising since it was previously observed that the path length changes very rapidly with initial latitude. Clearly the average group velocity must be much higher for the longer paths which are at a higher group veloc
latitude.

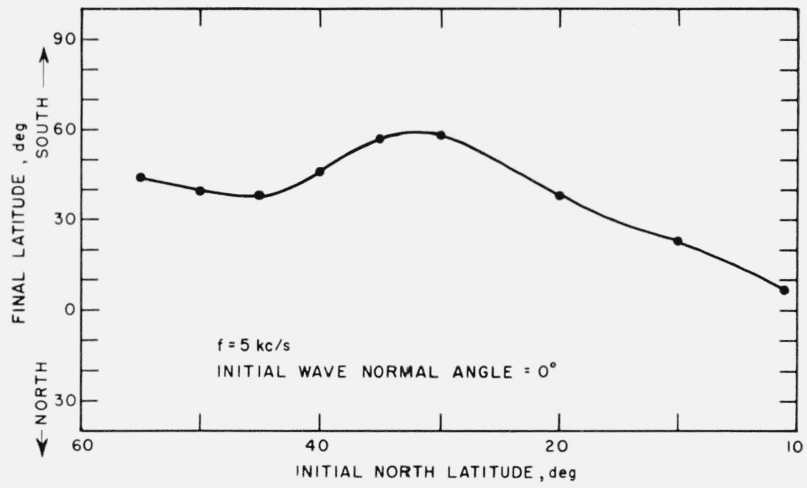

Figure 3(1). Final latitude versus initial latitude.

This plot of final latitude against initial value shows some focusing of rays in the region of $45^{\circ}$ and $33^{\circ}$ latitude.

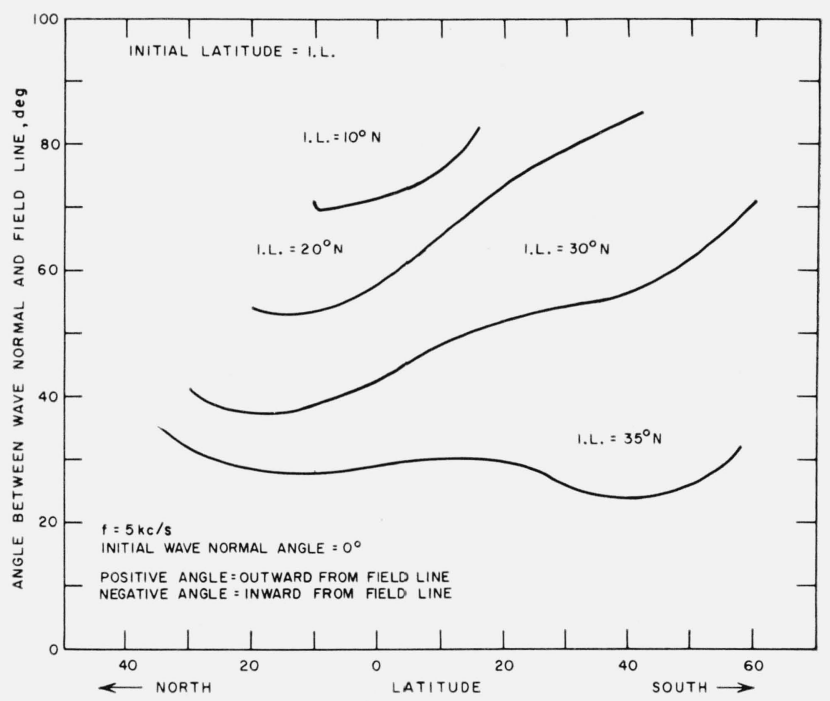

Figure $3(\mathrm{~m})$. Wave normal-field line angle along the path.

At initial north latitudes greater than $35^{\circ}$, the wave normal swings from the outside to the inside of the field line along the path. For initial north latitudes less than $35^{\circ}$, the wave normal swings farther away from the field line. 

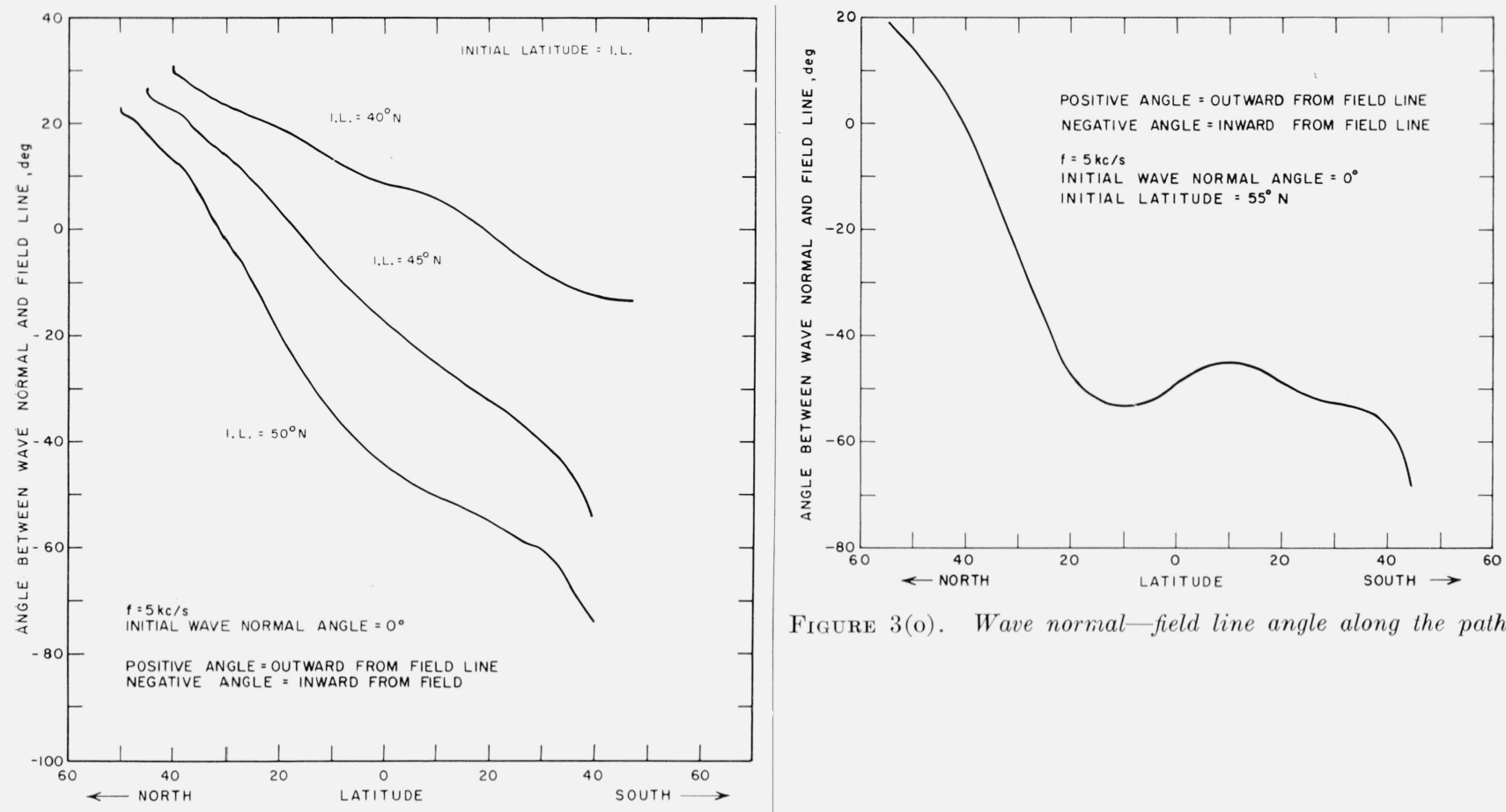

Figure 3(o). Wave normal-field line angle along the path.

Figure 3(n). Wave normal-field line angle along the path.

Figure 4(a). Ray path with initial wave normal angle $=10^{\circ}$.

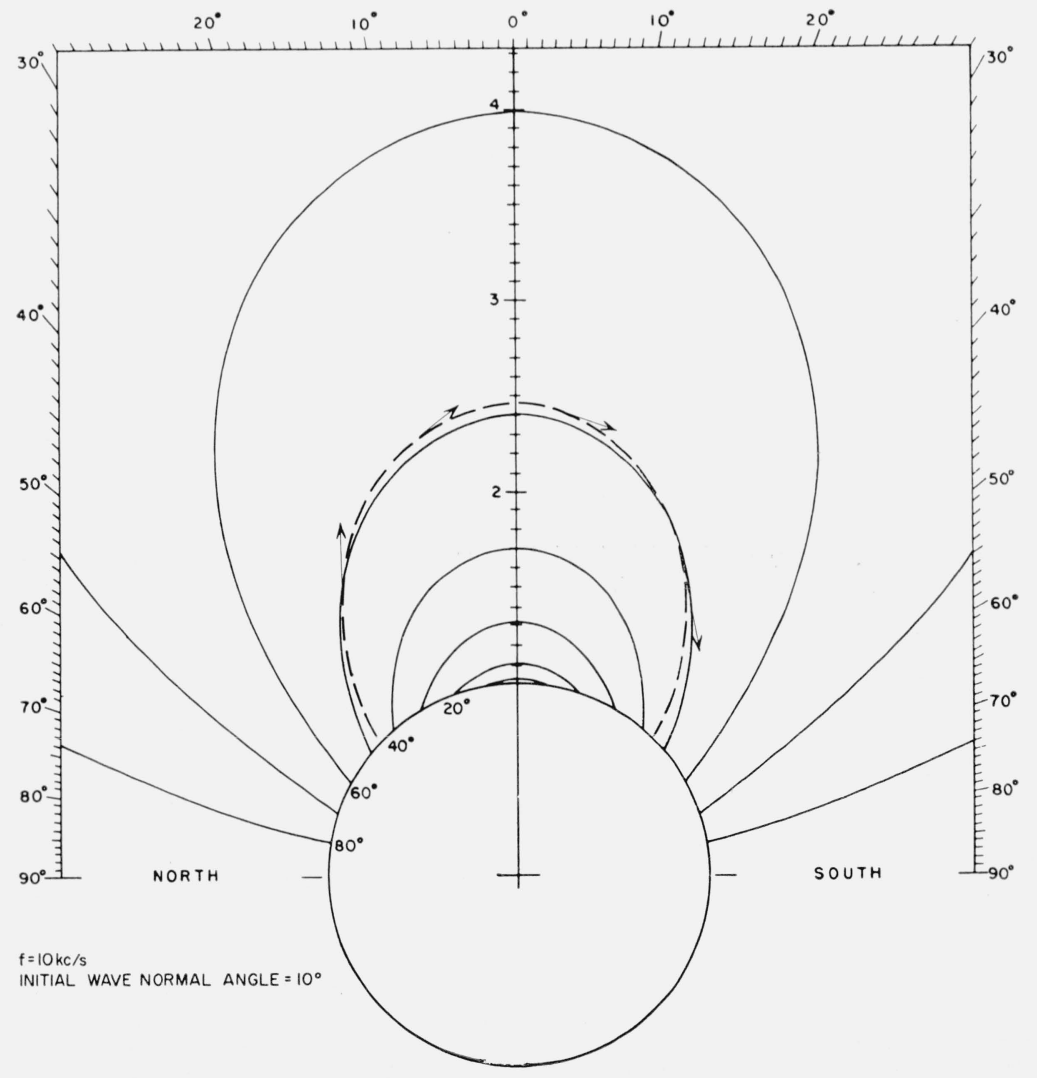




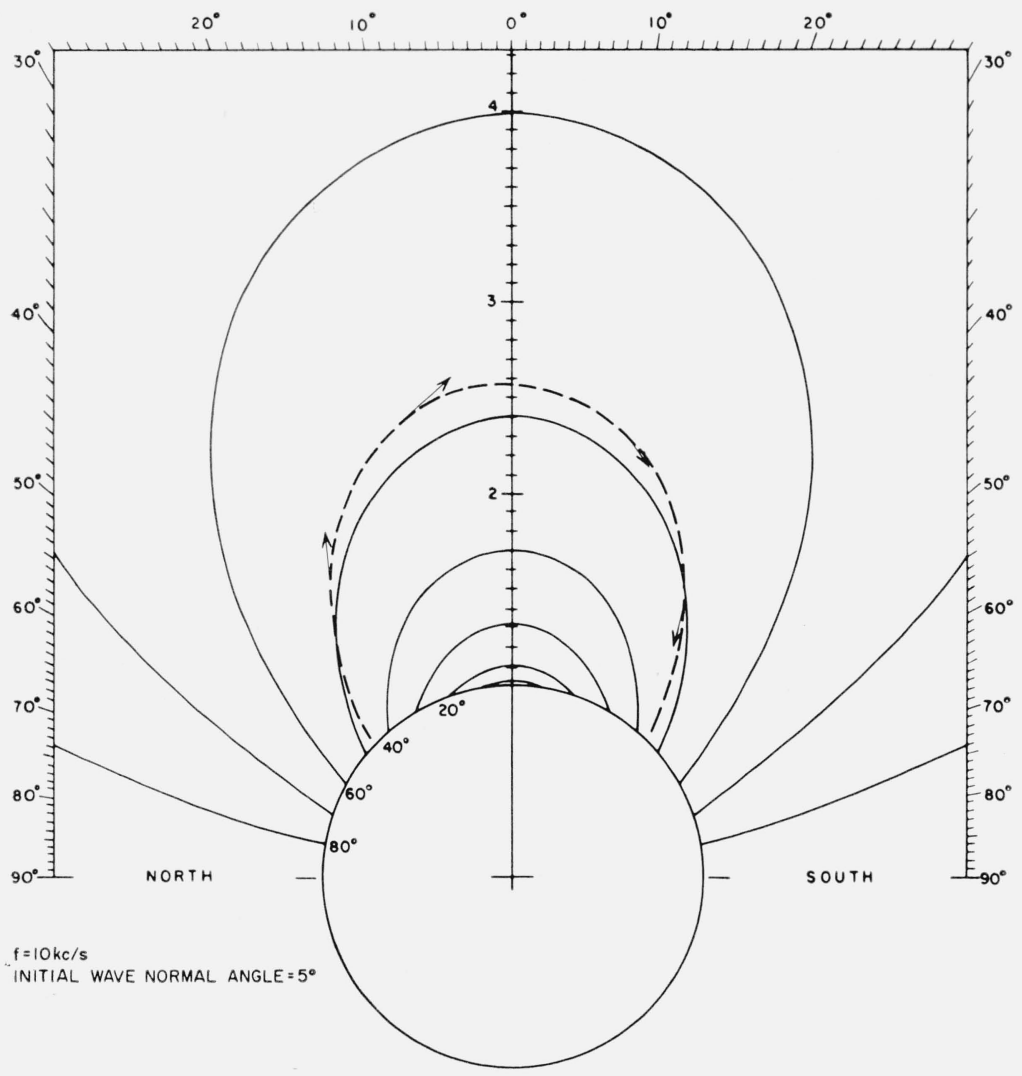

Figure 4(b). Ray path with initial wave normal angle $=5^{\circ}$

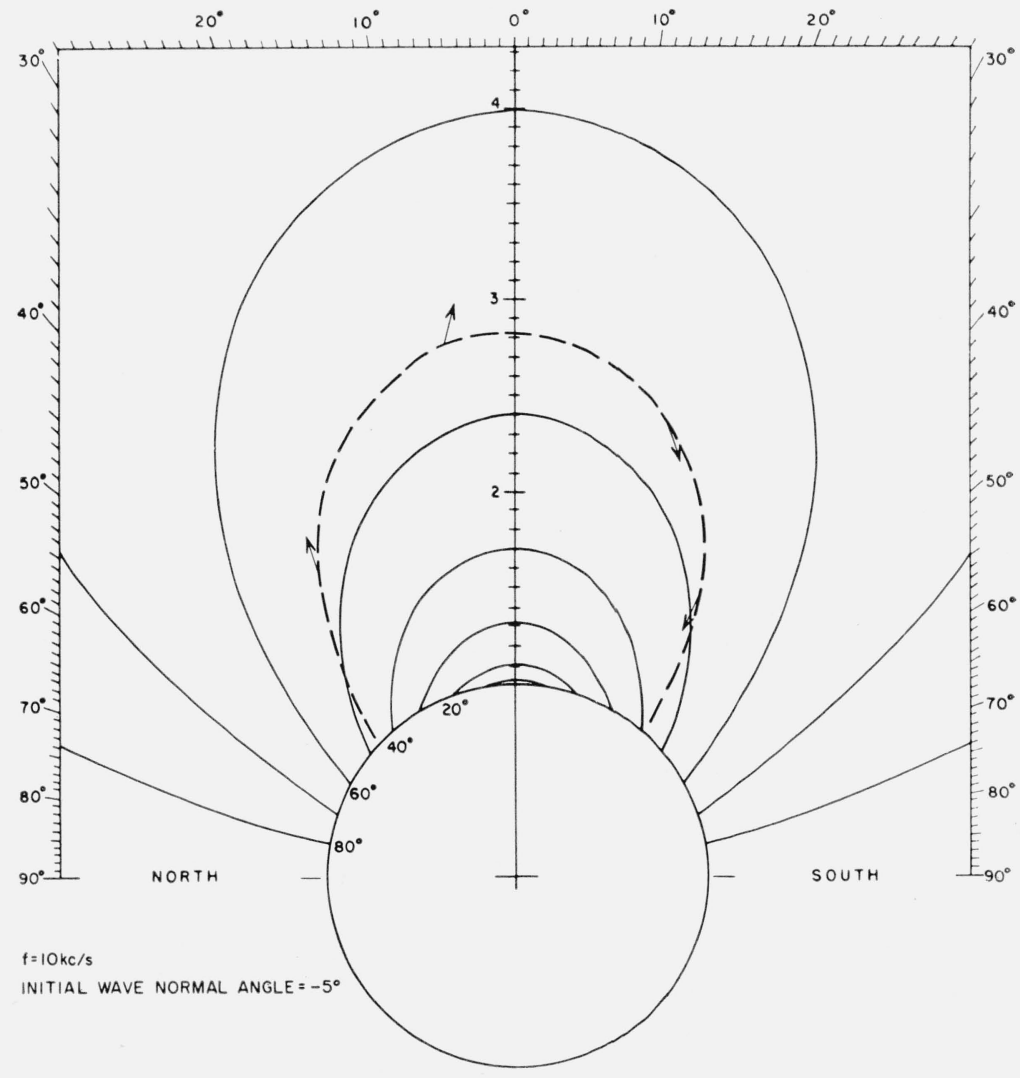




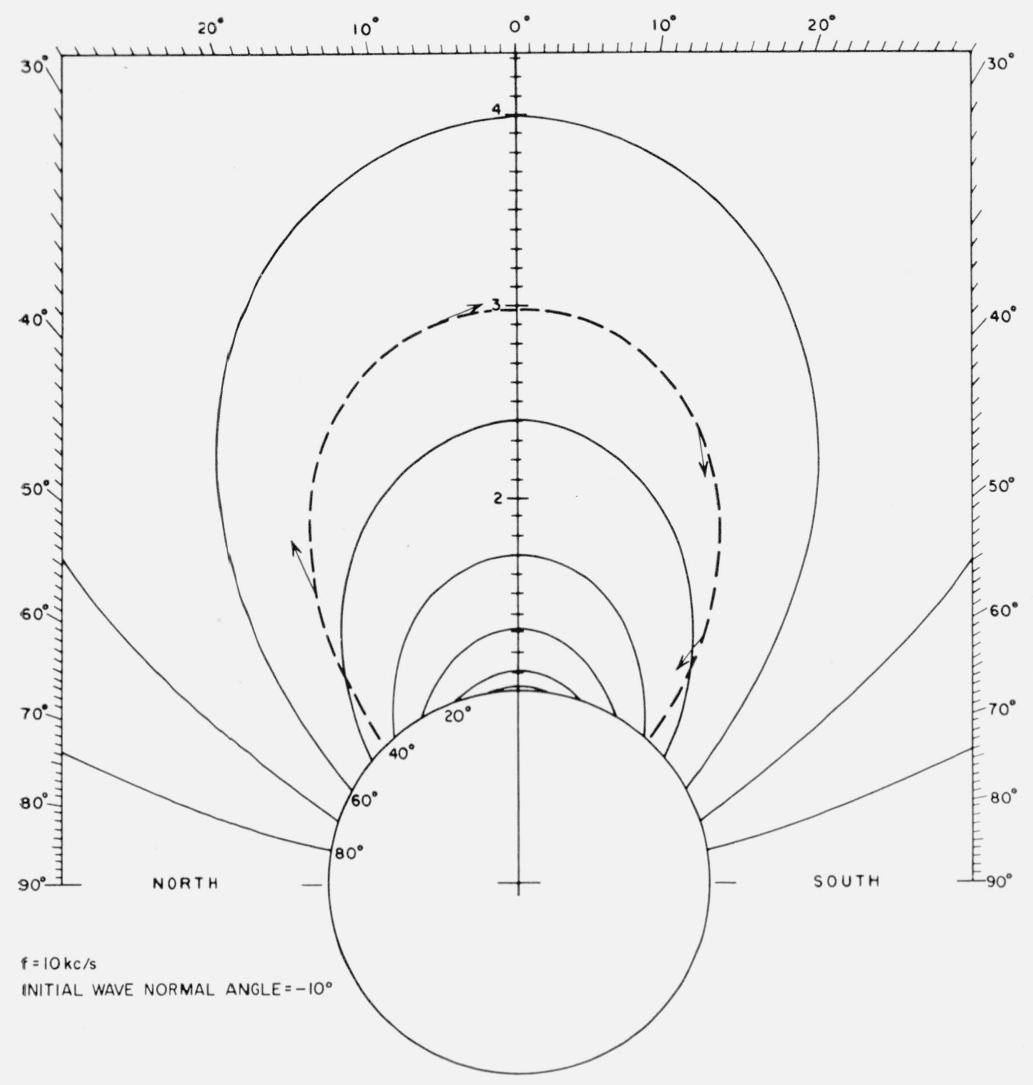

Figure 4(d). Ray path with initial wave normal angle $=10^{\circ}$.

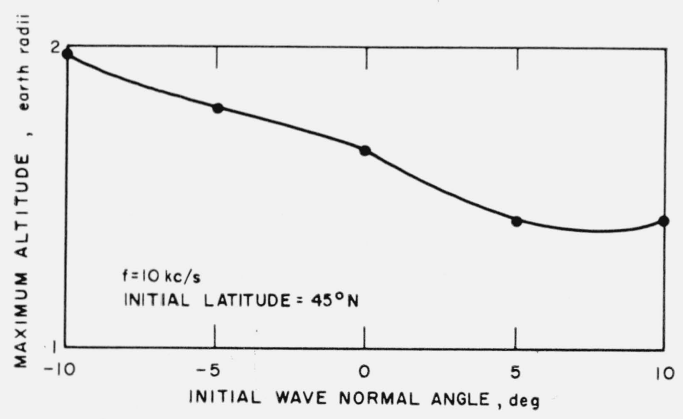

FIgure 4(e). Maximum altitude versus initial wave normal angle.

Maximum path height is seen to decrease with increasing wave normal angle until the wave reaches between $5^{\circ}$ and $10^{\circ}$.

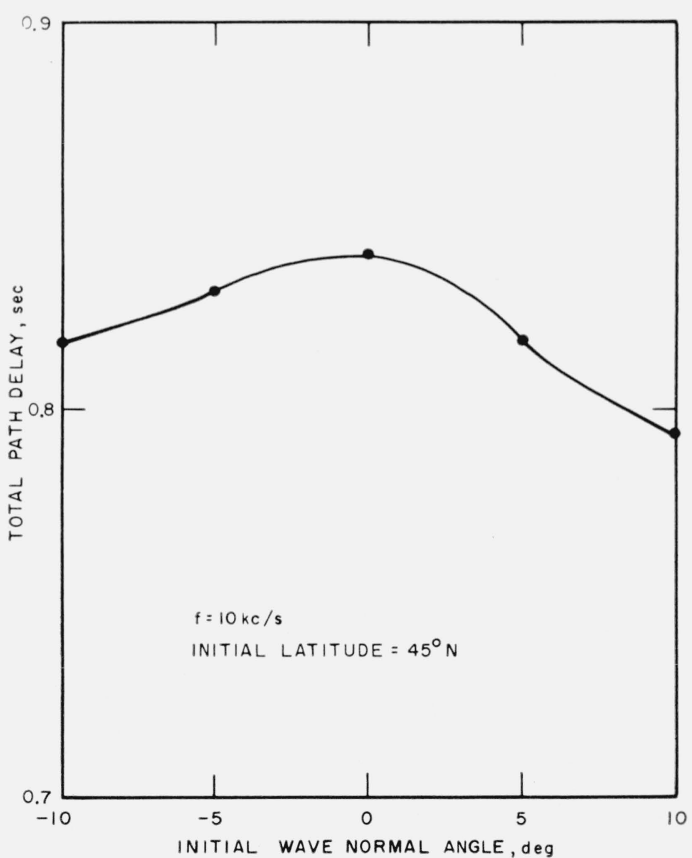

Figure 4(f). Total path delay versus initial wave normal angle.

The total time delay is also seen to remain roughly constant with changes in initial wave normal angle. This is again consistent with the idea of average group velocity being roughly proportional to path length. 


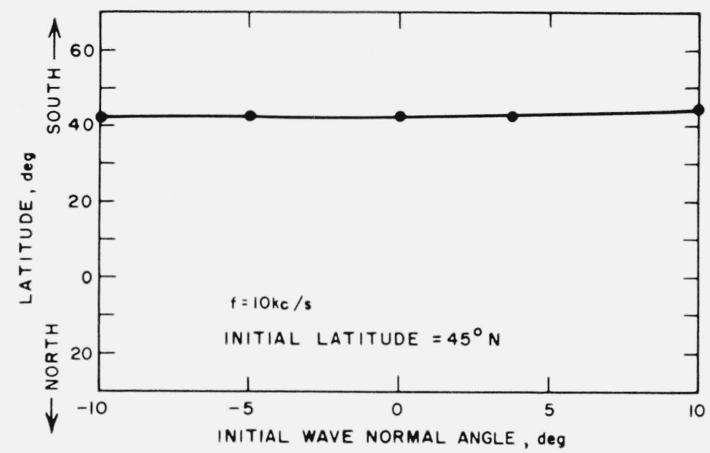

FIgure 4(g). Final latitude versus initial wave normal angle.

The final latitude is seen to remain constant with initial wave normal variation even though maximum path height changes.

Figure 5. Constant density model.

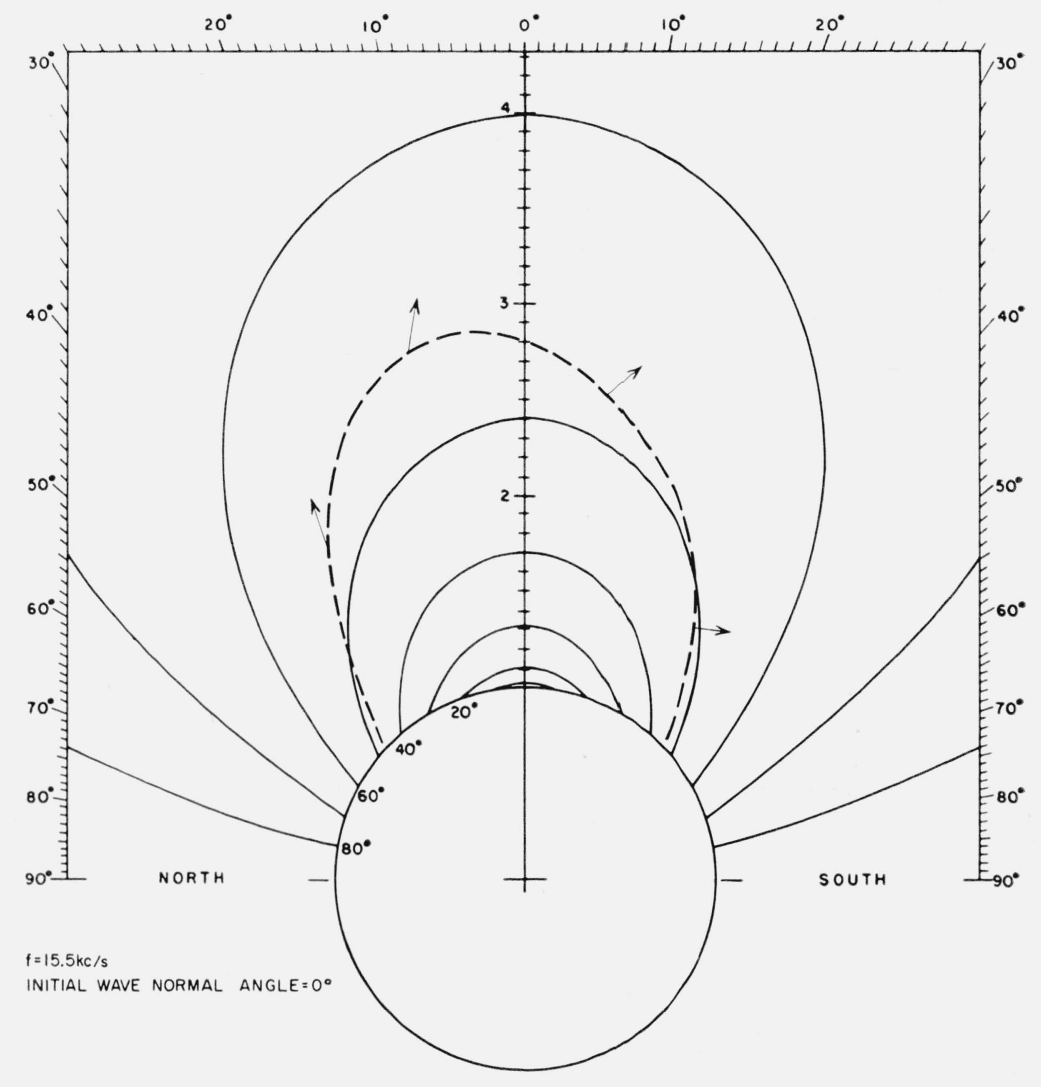




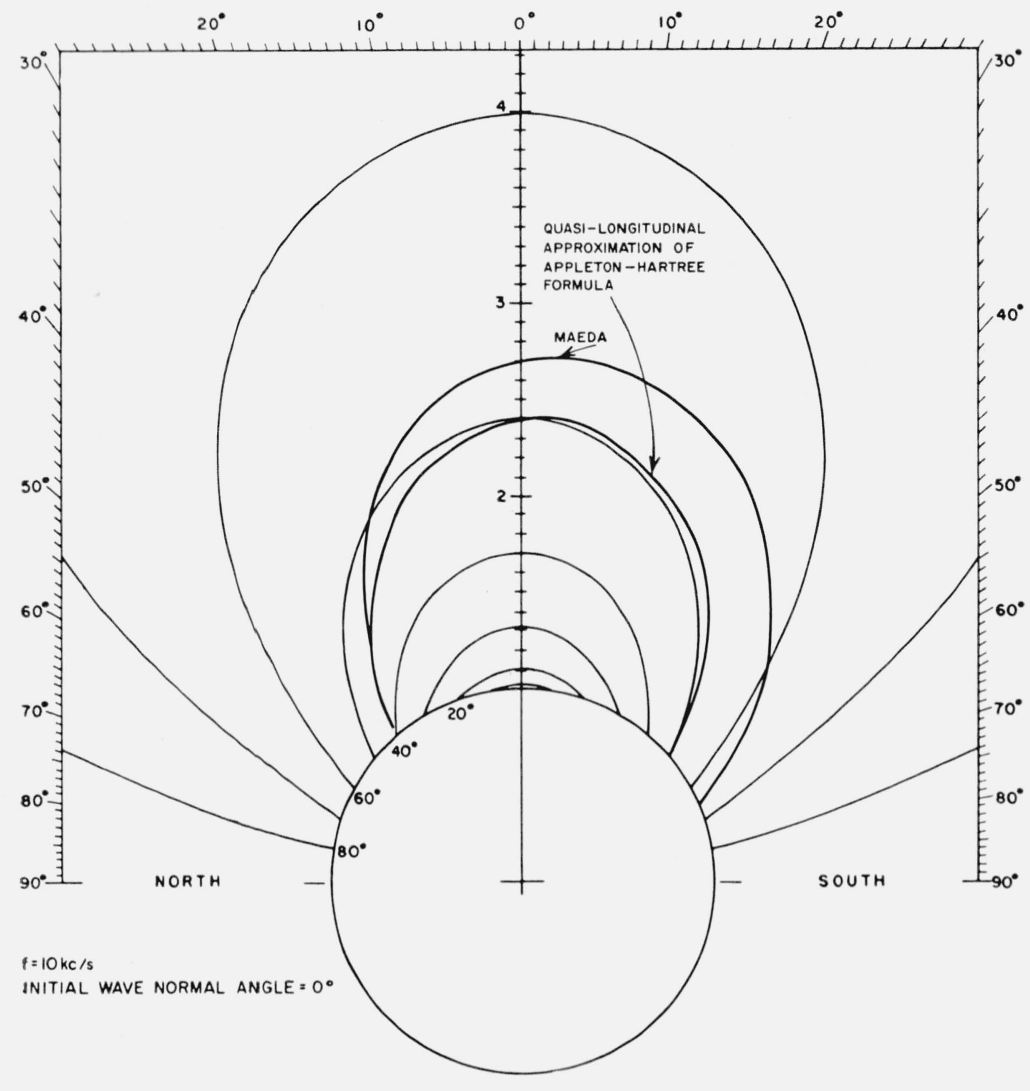

Figure 6. Comparison with Maeda's computation.

Figure 7. A field-alined column model.

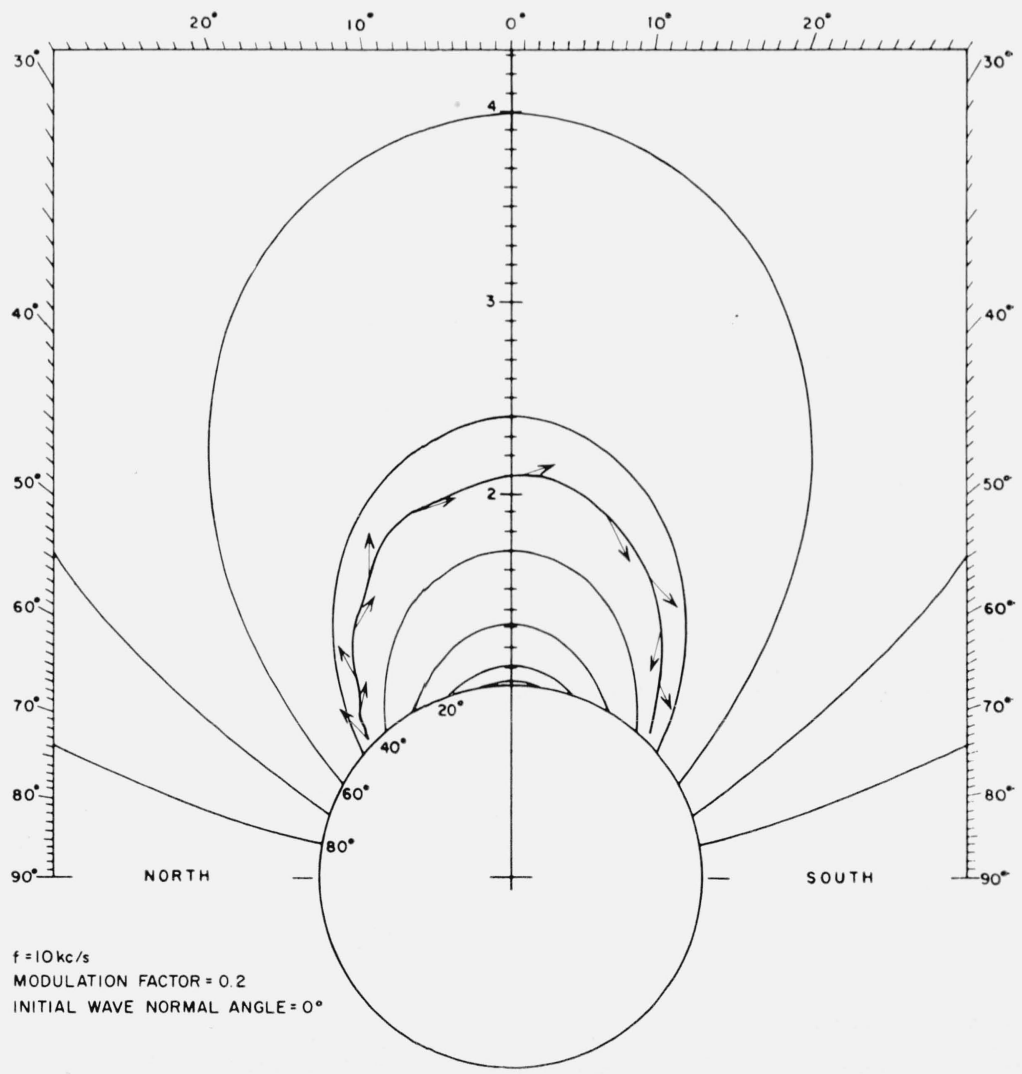




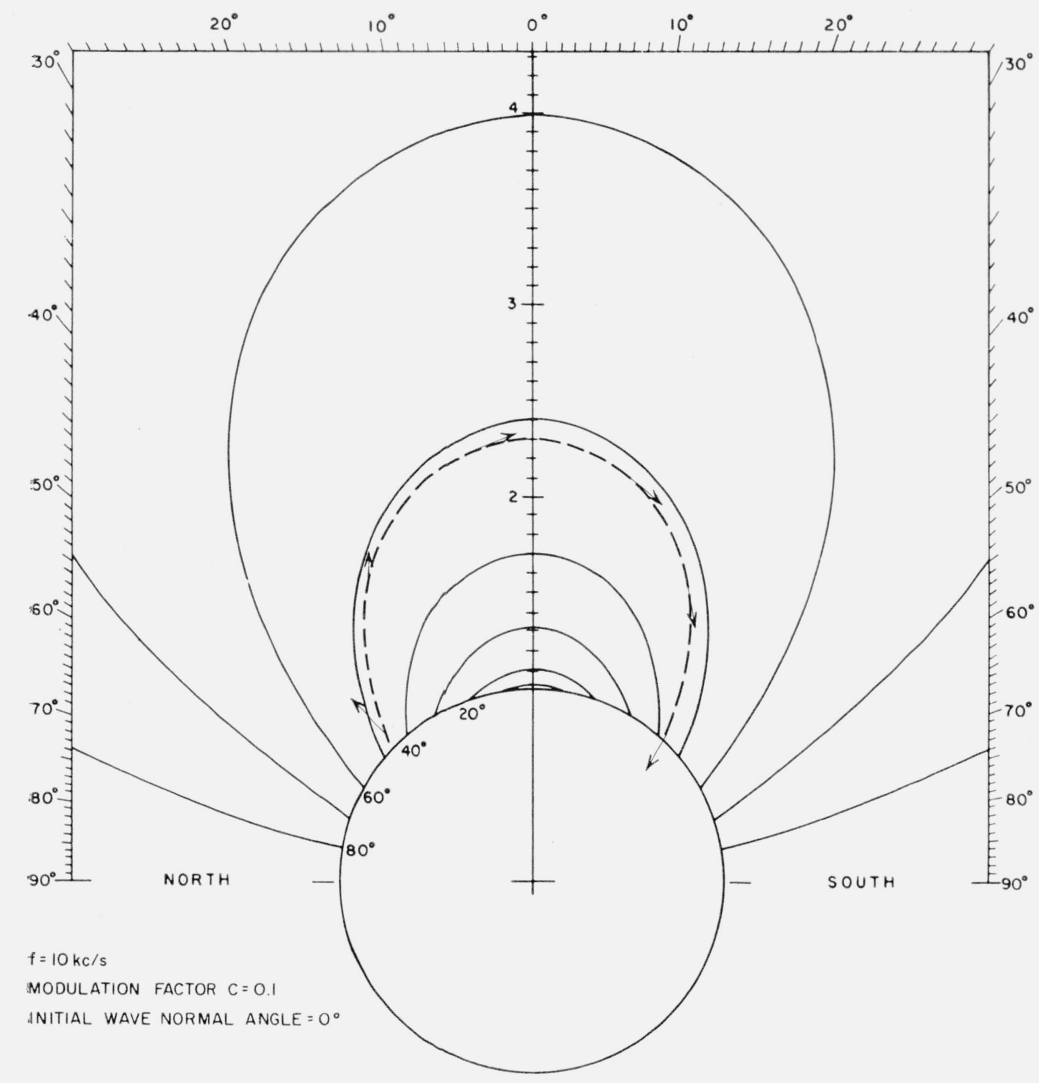

Figure 8. A field-alined column model.

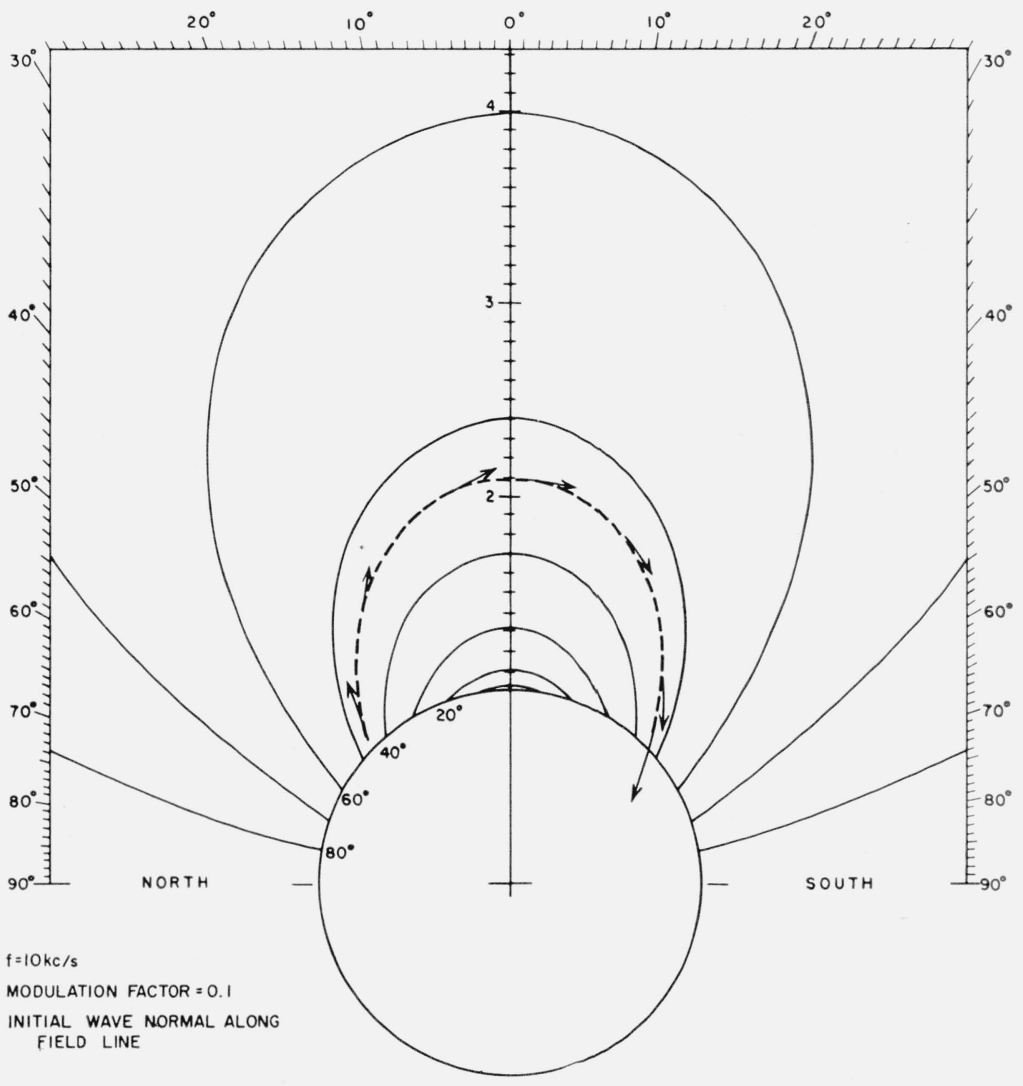




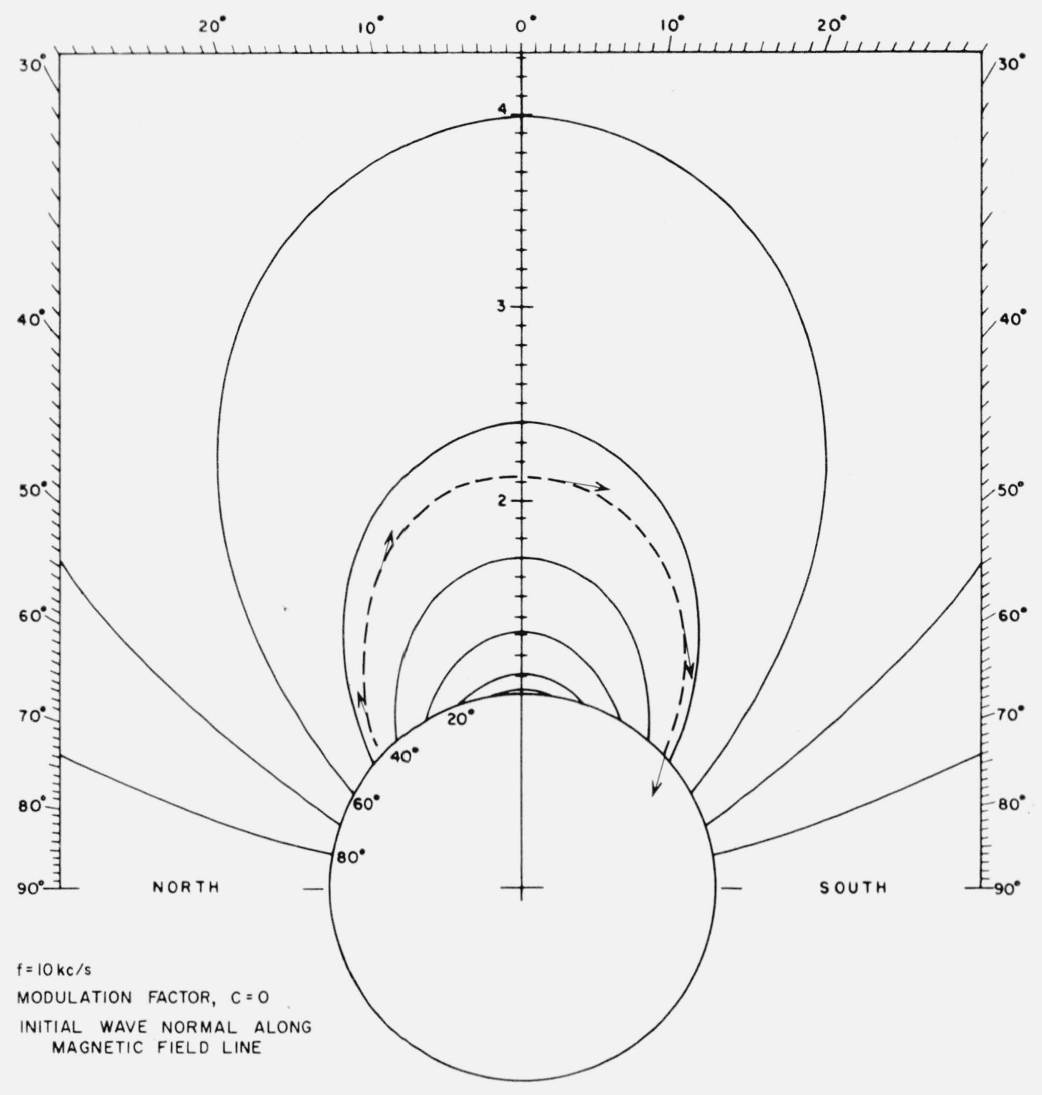

FIgURE 10. Ray path with initial wave normal along field line.

\section{References}

Haselgrove, J., Ray theory and a new method for ray tracing, Report of Phys. Soc. Conference on Physics of the Ionosphere, pp. 355-364, Cambridge (Sept. 1954).

Helliwell, R. A., and M. G. Morgan, Atmospheric whistlers, Proc. IRE 47, No. 2, 200-210 (Feb. 1959).

Maeda, K., and I. Kimura, A theoretical investigation on the propagation path of the whistling atmospherics, Rpt. Ionospheric Research Japan X, No. 3, 105-123 (Sept. 1956).
Ratcliffe, J. A., The magneto-ionic theory and its application to the ionosphere (Cambridge University Press, Cambridge, Mass., 1959).

Smith, R. L., R. A. Helliwell, and I. W. Yabroff, A theory of trapping of whistlers in field-aligned columns of enhanced ionization, J. Geophys. Research 65, No. 3, 815-823 (Mar. 1960).

Storey, L. R. O., Phil. Trans. Roy. Soc. [A] 246, pp. 113-141 (1953).

(Paper 65D5-155 